\title{
Assessment of CFD-based Response Surface Model for Ares I Supersonic Ascent Aerodynamics
}

\author{
Jeremy L. Hanke* \\ NASA Langley Research Center, Hampton, VA, 23681-2199
}

\begin{abstract}
The Ascent Force and Moment Aerodynamic (AFMA) Databases (DBs) for the Ares I Crew Launch Vehicle (CLV) were typically based on wind tunnel (WT) data, with increments provided by computational fluid dynamics (CFD) simulations for aspects of the vehicle that could not be tested in the WT tests. During the Design Analysis Cycle 3 analysis for the outer mold line (OML) geometry designated A106, a major tunnel mishap delayed the WT test for supersonic Mach numbers $(M)$ greater than 1.6 in the Unitary Plan Wind Tunnel at NASA Langley Research Center, and the test delay pushed the final delivery of the A106 AFMA DB back by several months. The aero team developed an interim database based entirely on the already completed CFD simulations to mitigate the impact of the delay. This CFD-based database used a response surface methodology based on radial basis functions to predict the aerodynamic coefficients for $M>1.6$ based on only the CFD data from both WT and flight Reynolds number conditions. The aero team used extensive knowledge of the previous AFMA DB for the A103 OML to guide the development of the CFD-based A106 AFMA DB. This report details the development of the CFD-based A106 Supersonic AFMA DB, constructs a prediction of the database uncertainty using data available at the time of development, and assesses the overall quality of the CFD-based DB both qualitatively and quantitatively. This assessment confirms that a reasonable aerodynamic database can be constructed for launch vehicles at supersonic conditions using only CFD data if sufficient knowledge of the physics and expected behavior is available. This report also demonstrates the applicability of non-parametric response surface modeling using radial basis functions for development of aerodynamic databases that exhibit both linear and non-linear behavior throughout a large data space.
\end{abstract}

\section{Nomenclature}

\begin{tabular}{|c|c|c|}
\hline \multicolumn{3}{|c|}{ Acronyms and Abbreviations } \\
\hline AFMA & $=$ & ascent force and moment aerodynamics \\
\hline CFD & $=$ & computational fluid dynamics \\
\hline CLV & $=$ & crew launch vehicle \\
\hline $\mathrm{CP}$ & $=$ & center of pressure \\
\hline $\mathrm{CxP}$ & $=$ & Constellation Program \\
\hline $\mathrm{C} 2 \mathrm{C}$ & $=$ & code-to-code \\
\hline DAC & $=$ & design analysis cycle \\
\hline DB & $=$ & database \\
\hline DBI & $=$ & database interpolation \\
\hline DBM & $=$ & database modeling \\
\hline DOF & & degree(s) of freedom \\
\hline EXP & & experimental \\
\hline FLT & & flight \\
\hline F\&M & & force(s) and moment(s) \\
\hline GN\&C & $=$ & Guidance, Navigation and Control \\
\hline GP & & gimbal point of the first stage \\
\hline ITAR & $=$ & International Traffic in Arms Regulations \\
\hline LaRC & & Langley Research Center \\
\hline
\end{tabular}

*Research Aerospace Engineer, Configuration Aerodynamics Branch, MS 499, Member AIAA.

1

American Institute of Aeronautics and Astronautics 


$\begin{array}{ll}\text { MI } & =\text { margin index } \\ \text { MRC } & =\text { moment reference center } \\ \text { OML } & =\text { outer mold line } \\ \text { RBF } & =\text { radial basis function } \\ \text { RS } & =\text { response surface } \\ \text { SBU } & =\text { sensitive but unclassified } \\ \text { UQ } & =\text { uncertainty quantification } \\ \text { UPWT } & =\text { Unitary Plan Wind Tunnel (NASA LaRC facility) } \\ \text { V\&V } & =\text { verification and validation } \\ \text { WT } & =\text { wind tunnel }\end{array}$
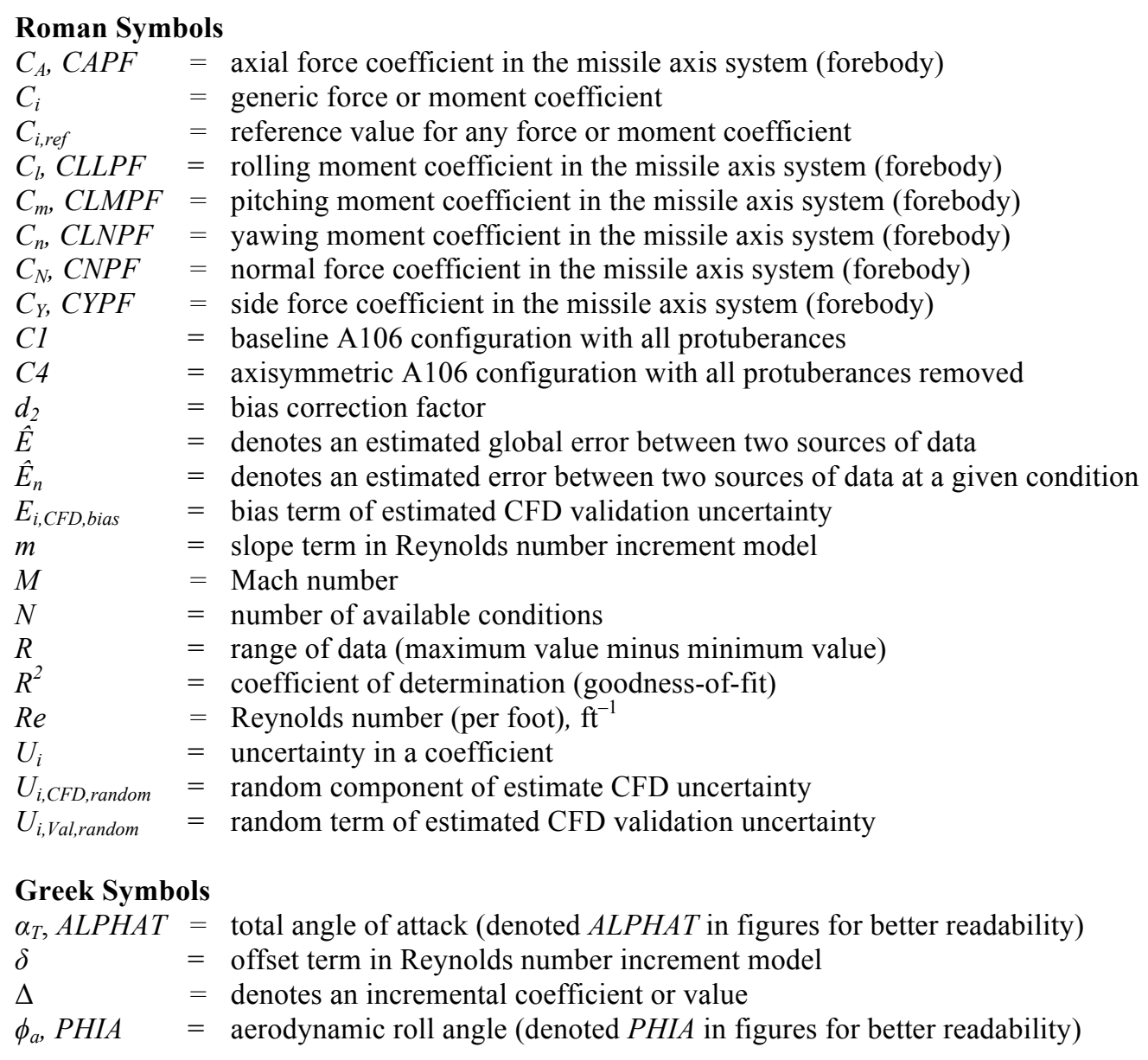

\section{Introduction}

$\mathrm{T}$ HE development and design process of the NASA crew launch vehicle (CLV), Ares I, required that the aerodynamics of the vehicles were adequately characterized for all phases of the flight envelope and reasonable estimates of the aerodynamic uncertainties were provided. One of the primary deliverables provided by the Ares I Aerodynamics Panel throughout the entire design process was the Ascent Force and Moment Aerodynamics (AFMA) Database (DB). This DB provided the six degree-of-freedom (DOF) aerodynamic force and moment (F\&M) coefficients for the CLV during nominal ascent flight from subsonic Mach numbers (M) through transonic and supersonic flight before stage separation (Mach numbers from 0.5 to around 5.5) for all total angles of attack below 10 degrees. The DB team constructed an AFMA DB from experimental data obtained in a suite of wind tunnels (WT) that spanned the entire Mach range for each major design analysis cycle (DAC) of the Ares I CLV. The team typically used NASA Langley Research Center's (LaRC) Unitary Plan Wind Tunnel (UPWT) as the primary wind tunnel for the supersonic $(M \geq 1.6)$ data for the AFMA DBs. The aero team also conducted computational fluid dynamics (CFD) simulations and used the CFD data to provide increments for elements that 
could not be reasonably tested in the wind tunnel, such as simulating the flight (FLT) Reynolds number (Re) environment. The CFD data were also used to provide data not practical via experimental means, such as surface pressure data on the surface of the CLV and lineloads, and to provide quick turnaround databases and increments for small geometry changes. The CFD best practices developed by the Aero Panel for Ares simulations dictated that all CFD solutions be obtained before the wind tunnel data. ${ }^{1}$

The CLV outer mold line (OML) geometry for DAC -3 was designated A106, and the aero team again planned and conducted a wind tunnel experiment in the UPWT (titled UPWT Tests 1972 and 1843) to acquire the supersonic data for the AFMA DB. ${ }^{2}$ Early on during the test, a major mechanical failure in the tunnel created a delay of over one month. This delay significantly impacted the project timeline because numerous analyses depend on receiving the AFMA DB. The aero team constructed an interim AFMA DB for $M>1.6$ using only the available CFD simulations to mitigate the effects of this delay. The aero team used a non-parametric response surface (RS) generation algorithm based on radial basis functions (RBFs) to construct the interim DB based on the available CFD solutions. RBF methodology allowed the team to tailor the final database to model the behavior expected based on previous AFMA DB data for the A103 OML. Several months after the CFD-based AFMA DB was released, the aero team developed and released the final A106 AFMA DB constructed from the UPWT Test 1972/1843 data. For convenience, this paper will refer to the initial DB as the CFD-based Supersonic A106 AFMA DB and the final DB as the WT-based Supersonic A106 AFMA DB, or just the CFD-based and WT-based DBs for short.

The purpose of this report is to present the methodology used to develop the CFD-based DB, develop a model for the uncertainty in the CFD-based DB based on data that were available at the time the CFD-based DB was developed, and assess the adequacy and accuracy of the CFD-based DB relative to the WT-based DB by comparing the results from each DB. Section I gives a brief introduction to the Ares I CLV, the available CFD solutions for the A106 OML, and response surface generation using radial basis functions. Section II details the methodology used to construct the CFD-based DB, and Section III discusses uncertainty modeling for the CFD-based DB. Section IV provides a brief overview of the final WT-based DB development and uncertainty. Section V compares the two databases, both qualitatively and quantitatively, to assess the quality and accuracy of the CFD-based DB relative to the final WT-based DB. Finally, some conclusions are presented in Section VI.

\section{A. The Ares I CLV}

Ares I was an in-line, two-stage, crew launch vehicle under development by NASA under the Constellation Project (CxP). The Ares I aerodynamics team, spanning several NASA centers, conducted extensive aerodynamic testing and computational simulations in support of the overall vehicle integration effort. ${ }^{3-9}$ Figure 1 shows the A106 OML for the vehicle along with definitions for the coordinate axis systems, aerodynamic force and moment coefficients, and flow angles. All force and moment coefficients in this report are forebody coefficients, meaning the forces on the base of the vehicle have been removed, and are reported with the moment reference center (MRC) at the first stage gimbal point (GP), which is approximately the origin of the axes systems in Figure 1. Reference 10, which summarizes the uncertainty modeling for the WT-based DB, details the effect of the MRC on uncertainty.

The Ares I CLV, including its predicted performance and certain other features and characteristics, has been defined by the U.S. Government to be sensitive but unclassified (SBU). Information deemed to be SBU requires special protection and may not be disclosed to an international audience, such as the audience present at the 2011 AIAA Applied Aerodynamics Conference. To comply with SBU restrictions, details such as axis scales have been removed from some figures in this paper and some results are presented in percent of reference values. It is the opinion of the author that these alterations do not invalidate the technical content and impact of this analysis. Whenever practical, the same grid scales were used for normal and side force coefficient and pitching and yawing moment so that the reader can infer the relative magnitudes of those coefficients. 


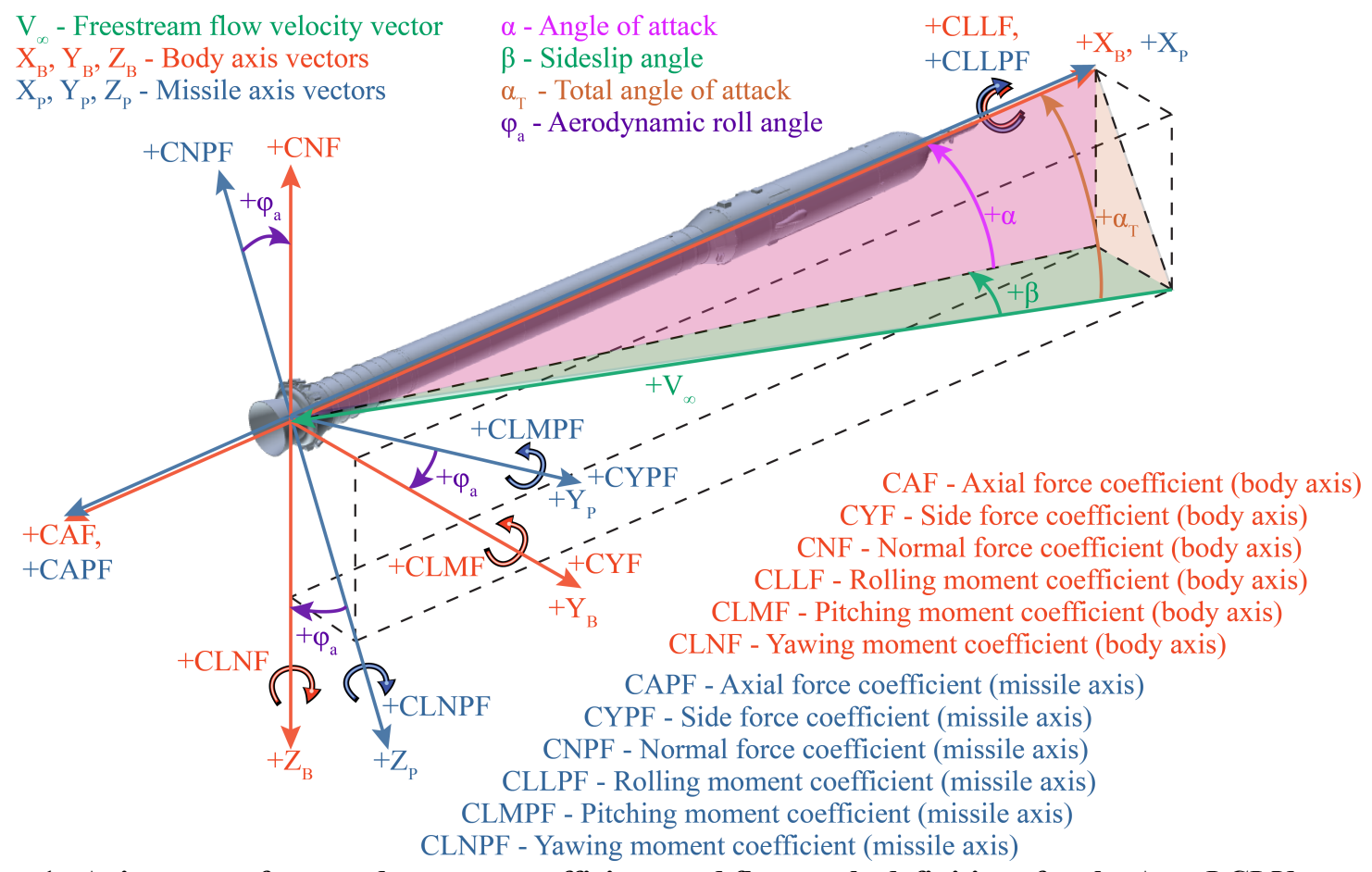

Figure 1. Axis system, force and moment coefficient, and flow angle definitions for the Ares I CLV.

\section{B. Summary of A106 CFD Data}

CFD simulations were a crucial aspect of the aerodynamic analyses performed by the Ares I aero team throughout the design process. CFD was used in numerous roles, such as supporting experimental data from wind tunnel tests and providing detailed information or simulating conditions not feasible experimentally. This paper does not describe the CFD analyses in depth; details on the CFD efforts for Ares I are available elsewhere. ${ }^{1,6-9}$ The aero team used two CFD codes to support the A106 AFMA DB: USM3D, which is an unstructured, tetrahedral, cellcentered, finite volume solver, ${ }^{11}$ and OVERFLOW, which is a structured, overset multiblock grid solver. ${ }^{12}$ OVERFLOW was considered the primary code for subsonic conditions, and USM3D was the primary code for transonic and supersonic computations. Each code repeated selected conditions from the other code's primary regime for code-to-code comparisons. This paper does not present any OVERFLOW solutions because USM3D was the primary code for $M>1.6$, and the DB team used only USM3D solutions to construct the CFD-based DB. Section III contains some discussion of code-to-code differences, and more details about the code-to-code comparisons from earlier Ares I DACs are reported in reference 13.

There were a total of 221 CFD solutions from USM3D for the A106 OML at the WT Re available when the CFD-based DB was developed, and 245 such solutions at FLT Re. Of these solutions, 104 WT Re and 77 FLT Re solutions corresponded to Mach numbers greater than or equal to 1.6. The UQ team requested an additional eight solutions (four for $M \geq 1.6$ ) at randomly chosen conditions throughout the domain for assessment of the CFD-based AFMA DB. Table 1 below lists the WT Re CFD simulations available for $M \geq 1.6$, and Table 2 lists the FLT Re solutions. The aero team partially designed the WT Re CFD matrix with response surface generation in mind, although the CFD team added numerous conditions to address other needs. The FLT Re matrix was primarily designed to address needs other than RS generation. The DB team designed the WT Re CFD matrix using general conceptual ideas from design of experiments (DOE), though the final matrix is not any particular DOE design. At 0 degrees total angle of attack, the aerodynamic roll angle is undefined by definition. For convenience, the team assumed an aerodynamic roll angle of 0 degrees for all CFD solutions acquired at total angle of attack of 0 degrees, but any other aerodynamic roll angle could have been chosen. 
Table 1. WT Reynolds number CFD solutions available for $M \geq 1.6$

\begin{tabular}{|c|c|c|}
\hline Mach Number & Total Angle of Attack & Aerodynamic Roll Angle \\
\hline \multirow{6}{*}{1.6} & $0^{\circ}$ & $0^{\circ}\left(\phi_{a}\right.$ undefined for $\left.\alpha_{T}=0^{\circ}\right)$ \\
\hline & $2^{\circ}$ & $0^{\circ}, 90^{\circ}, 180^{\circ}, 270^{\circ}$ \\
\hline & $4^{\circ}$ & $0^{\circ}, 30^{\circ}, 150^{\circ}, 180^{\circ}, 210^{\circ}, 345^{\circ}$ \\
\hline & $6^{\circ}$ & $90^{\circ}, 270^{\circ}$ \\
\hline & $7^{\circ}$ & $0^{\circ}$ to $330^{\circ}$ in $30^{\circ}$ increments \\
\hline & $8^{\circ}$ & $0^{\circ}$ to $330^{\circ}$ in $30^{\circ}$ increments, $340^{\circ}, 345^{\circ}, 350^{\circ}, 355^{\circ}$ \\
\hline \multirow{5}{*}{2.0} & $0^{\circ}$ & $0^{\circ}\left(\phi_{a}\right.$ undefined for $\left.\alpha_{T}=0^{\circ}\right)$ \\
\hline & $2^{\circ}$ & $0^{\circ}, 90^{\circ}, 180^{\circ}, 270^{\circ}$ \\
\hline & $4^{\circ}$ & $0^{\circ}, 60^{\circ}, 150^{\circ}, 180^{\circ}, 240^{\circ}, 345^{\circ}$ \\
\hline & $6^{\circ}$ & $90^{\circ}, 270^{\circ}$ \\
\hline & $8^{\circ}$ & $0^{\circ}, 90^{\circ}, 180^{\circ}, 270^{\circ}$ \\
\hline \multirow{5}{*}{3.0} & $0^{\circ}$ & $0^{\circ}\left(\phi_{a}\right.$ undefined for $\left.\alpha_{T}=0^{\circ}\right)$ \\
\hline & $2^{\circ}$ & $0^{\circ}, 90^{\circ}, 180^{\circ}, 270^{\circ}$ \\
\hline & $4^{\circ}$ & $0^{\circ}, 60^{\circ}, 150^{\circ}, 180^{\circ}, 240^{\circ}, 345^{\circ}$ \\
\hline & $6^{\circ}$ & $90^{\circ}, 270^{\circ}$ \\
\hline & $8^{\circ}$ & $0^{\circ}$ to $330^{\circ}$ in $30^{\circ}$ increments \\
\hline \multirow{5}{*}{4.5} & $0^{\circ}$ & $0^{\circ}\left(\phi_{a}\right.$ undefined for $\left.\alpha_{T}=0^{\circ}\right)$ \\
\hline & $2^{\circ}$ & $0^{\circ}, 90^{\circ}, 180^{\circ}, 270^{\circ}$ \\
\hline & $4^{\circ}$ & $0^{\circ}, 30^{\circ}, 60^{\circ}, 120^{\circ}, 150^{\circ}, 180^{\circ}, 210^{\circ}, 240^{\circ}, 300^{\circ}, 345^{\circ}$ \\
\hline & $6^{\circ}$ & $90^{\circ}, 270^{\circ}$ \\
\hline & $8^{\circ}$ & $0^{\circ}, 90^{\circ}, 180^{\circ}, 270^{\circ}$ \\
\hline
\end{tabular}

Table 2. FLT Reynolds number CFD solutions available for $M \geq 1.6$

\begin{tabular}{|c|c|c|}
\hline Mach Number & Total Angle of Attack & Aerodynamic Roll Angle \\
\hline \multirow{3}{*}{1.6} & $0^{\circ}$ & $0^{\circ}\left(\phi_{a}\right.$ undefined for $\left.\alpha_{T}=0^{\circ}\right)$ \\
\cline { 2 - 3 } & $4^{\circ}$ & $0^{\circ}, 60^{\circ}, 90^{\circ}, 120^{\circ}, 180^{\circ}, 240^{\circ}, 270^{\circ}, 300^{\circ}$ \\
\cline { 2 - 3 } & $8^{\circ}$ & $0^{\circ}$ to $330^{\circ}$ in $30^{\circ}$ increments \\
\cline { 2 - 3 } & $0^{\circ}$ & $0^{\circ}\left(\phi_{a}\right.$ undefined for $\left.\alpha_{T}=0^{\circ}\right)$ \\
\cline { 2 - 3 } & $4^{\circ}$ & $0^{\circ}, 30^{\circ}, 90^{\circ}, 120^{\circ}, 180^{\circ}, 210^{\circ}, 270^{\circ}, 300^{\circ}$ \\
\hline \multirow{3}{*}{2.0} & $8^{\circ}$ & $0^{\circ}, 30^{\circ}, 60^{\circ}, 90^{\circ}, 180^{\circ}, 270^{\circ}, 300^{\circ}, 330^{\circ}$ \\
\cline { 2 - 3 } & $0^{\circ}$ & $0^{\circ}\left(\phi_{a}\right.$ undefined for $\left.\alpha_{T}=0^{\circ}\right)$ \\
\hline \multirow{3}{*}{4.0} & $4^{\circ}$ & $0^{\circ}, 30^{\circ}, 90^{\circ}, 120^{\circ}, 180^{\circ}, 210^{\circ}, 270^{\circ}, 300^{\circ}$ \\
\hline \multirow{3}{*}{4.0} & $8^{\circ}$ & $0^{\circ}$ to $330^{\circ}$ in $30^{\circ}$ increments \\
\hline \multirow{3}{*}{4.5} & $0^{\circ}$ & $0^{\circ}\left(\phi_{a}\right.$ undefined for $\left.\alpha_{T}=0^{\circ}\right)$ \\
\hline & $4^{\circ}$ & $0^{\circ}, 30^{\circ}, 120^{\circ}, 210^{\circ}, 300^{\circ}$ \\
\hline & $0^{\circ}$ & $0^{\circ}\left(\phi_{a}\right.$ undefined for $\left.\alpha_{T}=0^{\circ}\right)$ \\
\hline & $4^{\circ}$ & $0^{\circ}, 90^{\circ}, 180^{\circ}, 270^{\circ}$ \\
\hline
\end{tabular}

\section{Response Surface Generation using Radial Basis Functions}

The intent of this paper is not to delve into the mathematics of radial basis function response surface methods (which are available elsewhere ${ }^{14-16}$ ); instead, this paper focuses on the application of RBF algorithms to a practical problem. However, a short discussion of RBF methodology is presented here for clarity. In general terms, radial basis function networks are analogous to solving a system of equations where each input data point has an "island of 
influence" on the global response. The relative size of each input point's "island" determines the importance of that input point to the final solution, allowing the user to weight the influence of each input point. A multidimensional response surface modeling software package (such as the NEAR RS software from Nielson Engineering and Research, Inc. ${ }^{16-19}$ used to develop the CFD-based A106 AFMA DB) constructs a global response surface across multiple dimensions based on a sparse set of input data.

There are several attractive features to using RBFs to generate the response surfaces for aerodynamic databases. Unlike parametric regression models, there are no requirements for the response surface to show linearity or parametric behavior in any dimension. Thus, radial basis functions can model extremely complex behavior. Additionally, RBFs do not require the input data to be regularly spaced in any dimension of the domain and can be updated easily as new data becomes available. In the author's experience, response surfaces constructed using RBF methodology are ideally suited to constructing aerodynamic databases that need to model complex behavior with only a relatively sparse set of input data available.

However, the features that make RBF methodology attractive for generating aerodynamic databases also lead to several of the corresponding challenges in a practical sense. Constructing an RS that adequately models the underlying data often requires extensive user input regarding the relative size of the "island of influence" for each data point. In the author's experience, this step of determining the proper "island of influence" for each input point is the single most important and difficult step in computing response surfaces using RBFs. Therefore, the author has found that this methodology is most useful in instances where the general behavior of the output response variables across the domain is known to some extent, so that the user can iterate the algorithm until the RS models the expected behavior. The CFD-based DB presented here was exactly such a circumstance, since the Ares I aero team had extensive knowledge of the aerodynamics of the Ares I CLV from the analyses performed during earlier design iterations. The A106 AFMA DB was expected to show similar general trends to the previous data. In such a situation, RBF methodologies are powerful tools that model the underlying data based on a reasonably sparse set of input data points.

\section{Development of the CFD-based Supersonic A106 AFMA DB}

The aero team developed the CFD-based Supersonic A106 AFMA DB using the NEAR RS software package. The objectives of the aero team for the CFD-based DB were to match the CFD data as closely as possible, to show proper physical behavior (aerodynamic coefficients show periodicity in $\phi_{a}$, etc), and to match the behavior expected based on data from previous design cycles. Discontinuities and large local slopes in the database generally present a challenge to the control of the vehicle, so the aero team desired the smoothest possible response surface that fulfilled the above goals. The flexibility of RBF response surface generation meant that the DB development was an iterative process, with the primary trade-off being the global smoothness of the RS versus going through the input CFD data. The team used the RS identification module of NEAR RS to compute a global RS across the desired domain of Mach number, total angle of attack $\left(\alpha_{T}\right)$, and aerodynamic roll angle $\left(\phi_{a}\right)$. The global RS was then queried at a regular set of breakpoints for the independent variables. Finally, the team made some final minor adjustments to the queried RS to ensure mathematical consistency.

For the CFD-based DB, separate response surfaces were generated for the longitudinal coefficients $\left(C_{A}\right.$, $C_{N}$, and $\left.C_{m}\right)$ and the lateral-directional coefficients $\left(C_{Y}, C_{l}\right.$, and $\left.C_{n}\right)$ in the missile axis system because the lateral-directional coefficients show much more complex behavior than the longitudinal coefficients, especially versus aerodynamic roll angle. While the missile axis $C_{Y}$ and $C_{n}$ in the AFMA DB have little impact, $C_{l}$ was considered a key driver to the trajectory, guidance, and control simulations of the Ares I CLV. The aerodynamic $C_{l}$ for the Ares I CLV was generated primarily by the interaction of aerodynamic loads on the protuberances, and the aero team observed that the rolling moment exhibited complex behavior across the domain throughout the Ares I aerodynamic analyses. ${ }^{3}$ The longitudinal response surface calculation was more robust than the lateral-directional RS; slight changes to the parameters or removal of input points did not affect the final longitudinal coefficient RS, while such changes had major effects on the lateral-directional RS. The sensitivity to changes in parameters made computing the lateraldirectional RS while preserving all desired behaviors especially difficult.

The DB team used 104 WT Re CFD solutions for the development and analysis of the CFD-based DB. The actual NEAR RS input file, however, contained more than 104 input data points. The DB team computed a simple linear model to adjust the final WT-based A106 AFMA DB to simulate FLT Re conditions based on CFD data at WT and FLT Re. ${ }^{10}$ The DB Team also computed the inverse of that linear model and applied it to the A106 FLT Re CFD solutions to create "approximate" WT Re solutions. The general form of this correction is shown in Eq. (1).

$$
C_{i, W T \text { approx. }}=m C_{i, F L T}+\delta
$$


At any point in the domain where an actual WT Re CFD solution existed, the approximate solution from the FLT Re CFD was omitted. The rest of these approximate WT Re CFD solutions (33 total) were included in the NEAR RS input files.

Unlike many parametric methods, the user cannot specify parameters such as derivatives of the response surface at the domain edges using RBFs. Instead, the DB team created "ghost" data points to ensure proper trends near the domain boundaries using the teams' experience with the behavior of the AFMA DB for previous design iterations as a guide. The CFD data for Mach numbers less than 1.6 were used to ensure the proper behavior near Mach 1.6. Several ghost points were added at Mach 5.0 (and a few points at Mach 6.5 for the lateral-directional RS) to ensure the expected behavior near Mach 4.5. By definition, the aerodynamic coefficients must be periodic with aerodynamic roll angle (since $\phi_{a}=-30$ degrees is the same as 330 degrees, etc.), so the team populated ghost data for aerodynamic roll angles from -90 to 0 degrees and 360 to 450 degrees using this periodicity. The data from the single CFD solution acquired at a total angle of attack of 0 degrees were extended across the entire aerodynamic roll angle domain at every 30 degrees because aerodynamic roll angle is undefined at zero degrees. The team also added interpolated data points at 0 degrees total angle of attack for the nominal DB Mach number breakpoints where no CFD data were available $(1.8,2.5$, and 3.5). The DB team reflected the data from 0 to 4 degrees total angle of attack about 0 degrees to correctly model coefficient behavior near $\alpha_{T}=0$ degrees. For the same reasons, the team created ghost points at 10 degrees total angle of attack by extrapolating the available CFD data. Finally, the team added eight points at $\alpha_{T}=6$ degrees for aerodynamic roll angles of 0 and 180 degrees approximated using a smoothing spline interpolation based on the A103 AFMA DB behavior at those conditions.

The final CFD-based Supersonic A106 AFMA DB closely matched the available CFD data. Table 3 provides the computed coefficient of determination $\left(R^{2}\right)$ statistics for the CFD-based DB evaluated at both the input CFD points and the DB assessment CFD points. The coefficient of determination is a standard statistical metric for evaluating goodness-of-fit and is often used for polynomial and other regression models. ${ }^{25} \mathrm{~A}$ value close to unity represents very little quantitative difference between the model and the input points, while a value less than 0.5 in general represents a poorer fit. The results in Table 3 clearly show that the CFD-based DB closely models the CFD data. The $R^{2}$ values for the lateral-directional coefficients indicate a somewhat poorer fit than for the longitudinal coefficients, but the high $R^{2}$ values still indicate good agreement between the CFD-based DB and the input CFD data. The very low $R^{2}$ value at the $\mathrm{DB}$ assessment points for $C_{Y}$ occurred because the predicted coefficient values at those conditions were small in magnitude, so the $R^{2}$ data are somewhat misleading for that case.

Table 3. Coefficient of determination $\left(R^{2}\right)$ statistics for the CFD-based DB evaluated at the 104 input WT Re CFD points and the four DB assessment points.

\begin{tabular}{|c|c|c|}
\hline Coefficient & DB Input Points $\boldsymbol{R}^{2}$ & DB Assessment Points $\boldsymbol{R}^{2}$ \\
\hline$C_{A}$ & 0.9993 & 0.9990 \\
\hline$C_{Y}$ & 0.9472 & 0.0126 \\
\hline$C_{N}$ & 0.9999 & 0.9998 \\
\hline$C_{l}$ & 0.9987 & 0.9130 \\
\hline$C_{m}$ & 1.0 & 0.9992 \\
\hline$C_{n}$ & 0.9903 & 0.8323 \\
\hline
\end{tabular}

The input file for the longitudinal coefficient RS had 774 input points, and the lateral-directional RS input file had 786 input points. The RS was queried at the nominal DB breakpoints in Mach number (1.6, 1.8, 2.0, 2.5, 3.0, $3.5,4.0$ and 4.5$)$, total angle of attack ( 0 to 8 degrees in one degree increments), and aerodynamic roll angle (0 to 360 degrees in 15 degree increments). The DB team made minor corrections to the data queried from NEAR RS to ensure exact data periodicity with aerodynamic roll angle and proper behavior at 0 degrees total angle of attack. Figure 2 shows an isometric surface plot of the CFD-based A106 AFMA DB for $C_{l}$ at a total angle of attack versus Mach number and aerodynamic roll angle, with the corresponding original WT Re CFD data plotted as squares. It is clear from the figure that the CFD-based DB closely matches the available CFD data points and exhibits complex behavior with Mach number and aerodynamic roll angle. Figure 3 shows a similar plot for $C_{A}$, and Fig. 4 gives the same plot for $C_{N}$. 


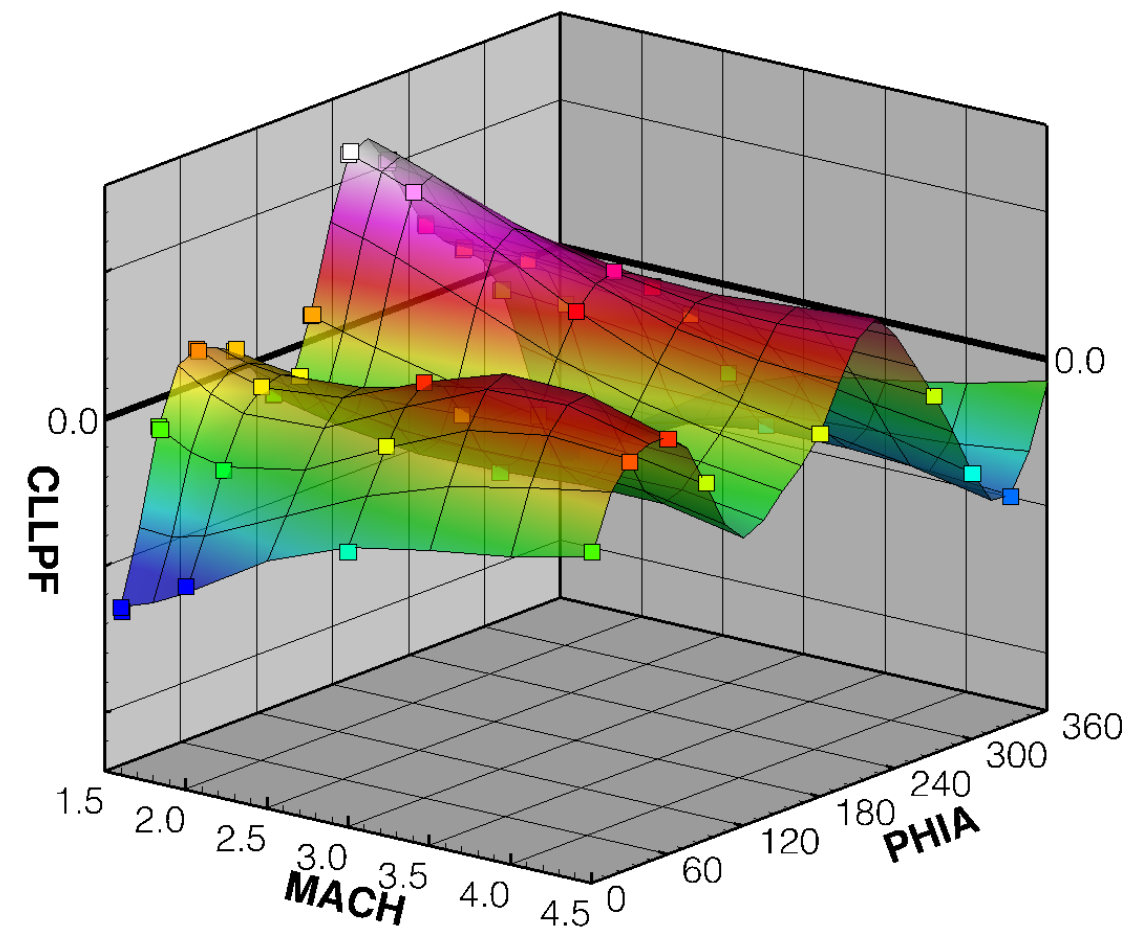

Figure 2. CFD-based Supersonic A106 AFMA DB and WT Re CFD data for $C_{l}$ at $\alpha_{T}=8$ degrees versus $M$ and $\phi_{a}$.

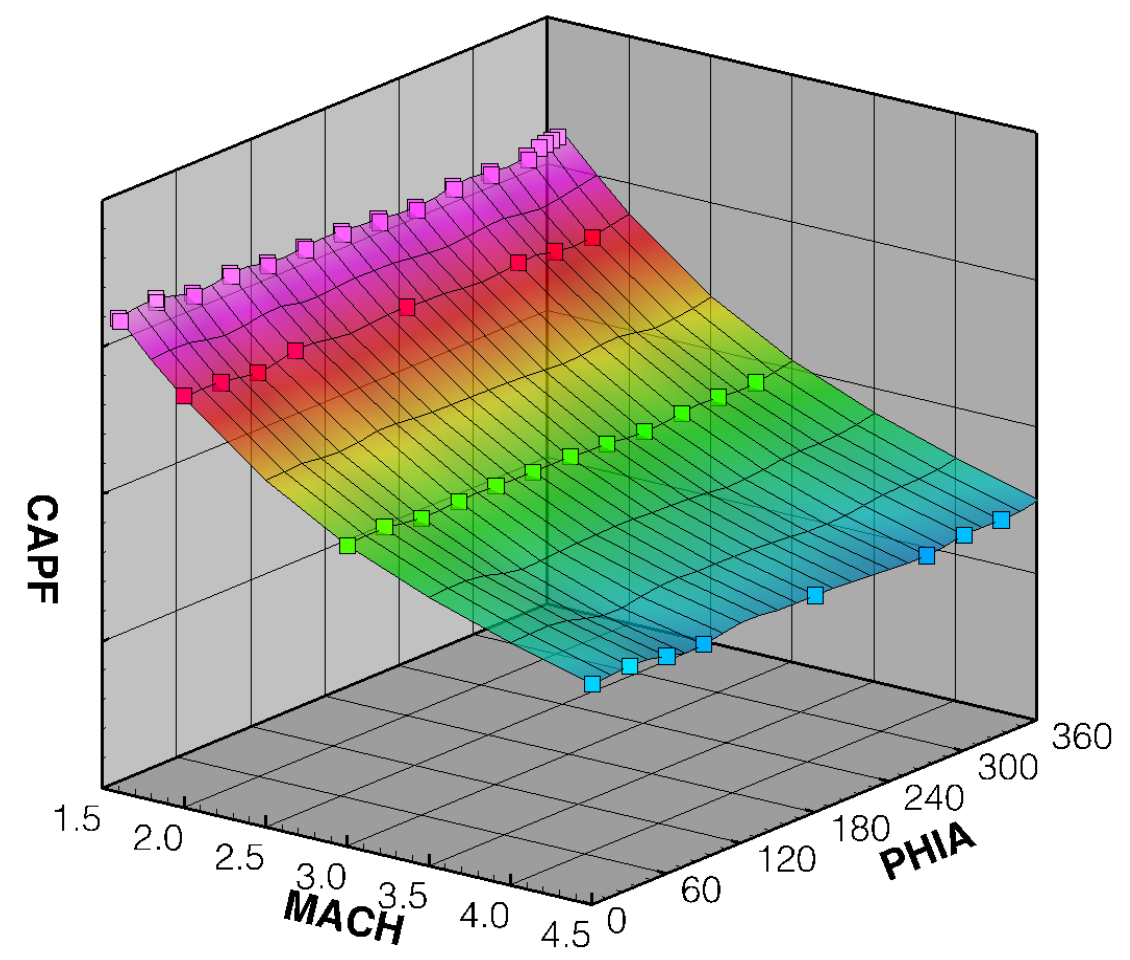

Figure 3. CFD-based Supersonic A106 AFMA DB and WT Re CFD data for $C_{A}$ at $\alpha_{T}=8$ degrees versus $M$ and $\phi_{a}$. 


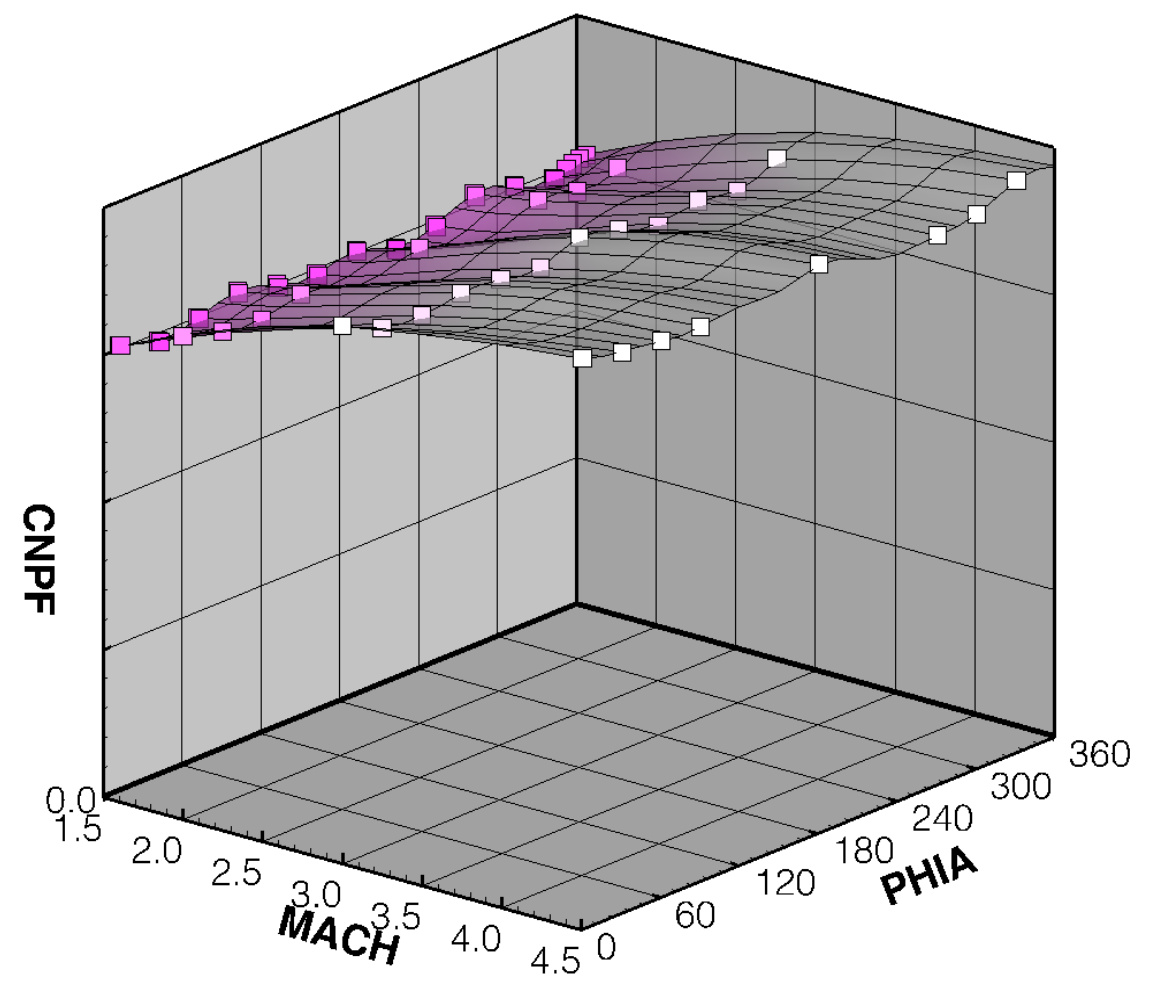

Figure 4. CFD-based Supersonic A106 AFMA DB and WT Re CFD data for $C_{N}$ at $\alpha_{T}=8$ degrees versus $M$ and $\phi_{a}$.

\section{Uncertainty Modeling for the CFD-based Supersonic A106 AFMA DB}

The Ares UQ team had not established a standard methodology for quantifying errors in CFD solutions when the CFD-based Supersonic A106 AFMA DB was developed; however, a reasonable estimate for the uncertainty of the CFD-based DB can be computed from information available at the time. The uncertainty model presented here for the CFD-based Supersonic A106 AFMA DB follows the general methodology used for the WT-based Supersonic A106 AFMA DB. ${ }^{10}$ The UQ team estimated uncertainties for significant sources of uncertainty in the database development process. In general, these sources can be split into three main categories: (1) input data uncertainty, (2) database modeling (DBM) uncertainty, and (3) database interpolation (DBI) uncertainty. For the CFD-based DB, the A106 CFD solutions are the input data, so the input data uncertainty is the uncertainty in the CFD solutions. The overall uncertainty model for the CFD-based DB is shown in Eq. (2). The UQ team assumed that each of the uncertainty sources was uncorrelated with the others. The CFD data uncertainty and the overall model have both a bias and random component. Some of the residuals included in the random uncertainty component may not actually be random errors, but the UQ team treated the residuals as random variations by pooling data across the domain.

$$
\begin{gathered}
U_{i, \mathrm{CFD} \mathrm{DB}, \text { random }}=\sqrt{U_{i, C F D, \text { random }}^{2}+U_{i, D B M}^{2}+U_{i, D B I}^{2}} \\
U_{i, \mathrm{CFD} D \mathrm{~B}, \text { bias }}=E_{i, C F D, \text { bias }}
\end{gathered}
$$

\section{A. A106 CFD Data Uncertainty}

Quantifying the uncertainty for a set of CFD solutions typically involves both a verification and validation (V\&V) phase (see Ref. 20-22 for further discussion of V\&V for CFD). Verification refers to the process of confirming that a computational model implementation accurately represents the underlying conceptual model and its solution, ${ }^{22}$ which for CFD codes typically includes order of accuracy tests, grid convergence tests, and comparisons with exact or highly accurate solutions. Validation refers to the process of determining if a simulation is an accurate representation of the intended real-world application ${ }^{22}$ and typically involves comparing the simulated results to wind tunnel and flight test data and evaluating the accuracy of the simulation. The uncertainty model 
developed by the UQ team for the input A106 CFD solutions is shown in Eq. (3) and reflects both aspects of V\&V. The model contains a term constructed from code-to-code comparisons $\left(U_{i, C 2 C}\right)$ as well as both bias $\left(E_{i, C F D, b i a s}\right)$ and random $\left(U_{i, \text { Val,random }}\right)$ validation uncertainty terms from comparison of CFD data to WT data from the earlier Ares I $\mathrm{DAC}$ on the $\mathrm{A} 103 \mathrm{OML}$. The $k_{M I}$ term in the model is a margin index added for conservatism because the CFD validation comparisons for the A106 OML may not be identical to the A103 comparisons. The UQ team selected a value of 1.15 for this term based on engineering judgment, which corresponds to increasing the estimated random validation uncertainty term by 15 percent.

$$
\begin{gathered}
U_{i, C F D, \text { random }}=\sqrt{U_{i, C 2 C}^{2}+\left(k_{M I} U_{i, \text { Val }, \text { random }}\right)^{2}} \\
U_{i, C F D, \text { bias }}=E_{i, C F D, \text { bias }}
\end{gathered}
$$

A classical solution verification analysis for a set of CFD solutions involves estimating the discretization error in the solutions through the rigorous use of grid refinement and metrics such as Richardson extrapolation. ${ }^{21}$ That type of detailed analysis for each set of CFD solutions is often difficult to perform in a fast-paced vehicle design project because of schedule and resource limitations. The general quality of the Ares I CFD solutions was confirmed throughout the DAC process through the establishment and revision of best practices, ${ }^{1,6}$ but the discretization errors for the A106 CFD solutions were not rigorously quantified. Instead, the UQ team merged code-to-code comparisons for the A106 OML with previously presented evaluations of the code-to-code differences in the CFD solutions ${ }^{13}$ to establish a surrogate estimate of the discretization error in the CFD solutions. The detailed methodology for computing the code-to-code term will only be summarized here. At all conditions with CFD solutions available from more than one CFD code, the UQ team computed the range of all available data for each coefficient by finding the absolute value of the difference between the maximum and minimum values. The UQ team then computed a robust estimator of the standard deviation by dividing each range by a statistical bias factor $\left(d_{2}\right)$ that depends only on the number of solutions used to compute each range ${ }^{23}$ After examining the normalized range $\left(R / d_{2}\right)$ data, the UQ team determined that the code-to-code differences for $M \geq 1.6$ were primarily dependent on the total angle of attack and pooled the normalized ranges across all of the other variables. Additionally, the UQ team pooled the data from the A101, A103, and A106 OMLs and for simulations at both WT and FLT Reynolds numbers. Finally, the UQ team estimated the code-to-code uncertainty term in Eq. (3) by determining a reasonably smooth curve that bounded all of the normalized range data. Figure 5 shows the normalized ranges and the estimated code-to-code uncertainty. Each data point in the figure corresponds to the normalized range of data from all codes at a unique combination of OML and Re (i.e., "A101 WT" denotes the data from the A101 OML at WT Re). 


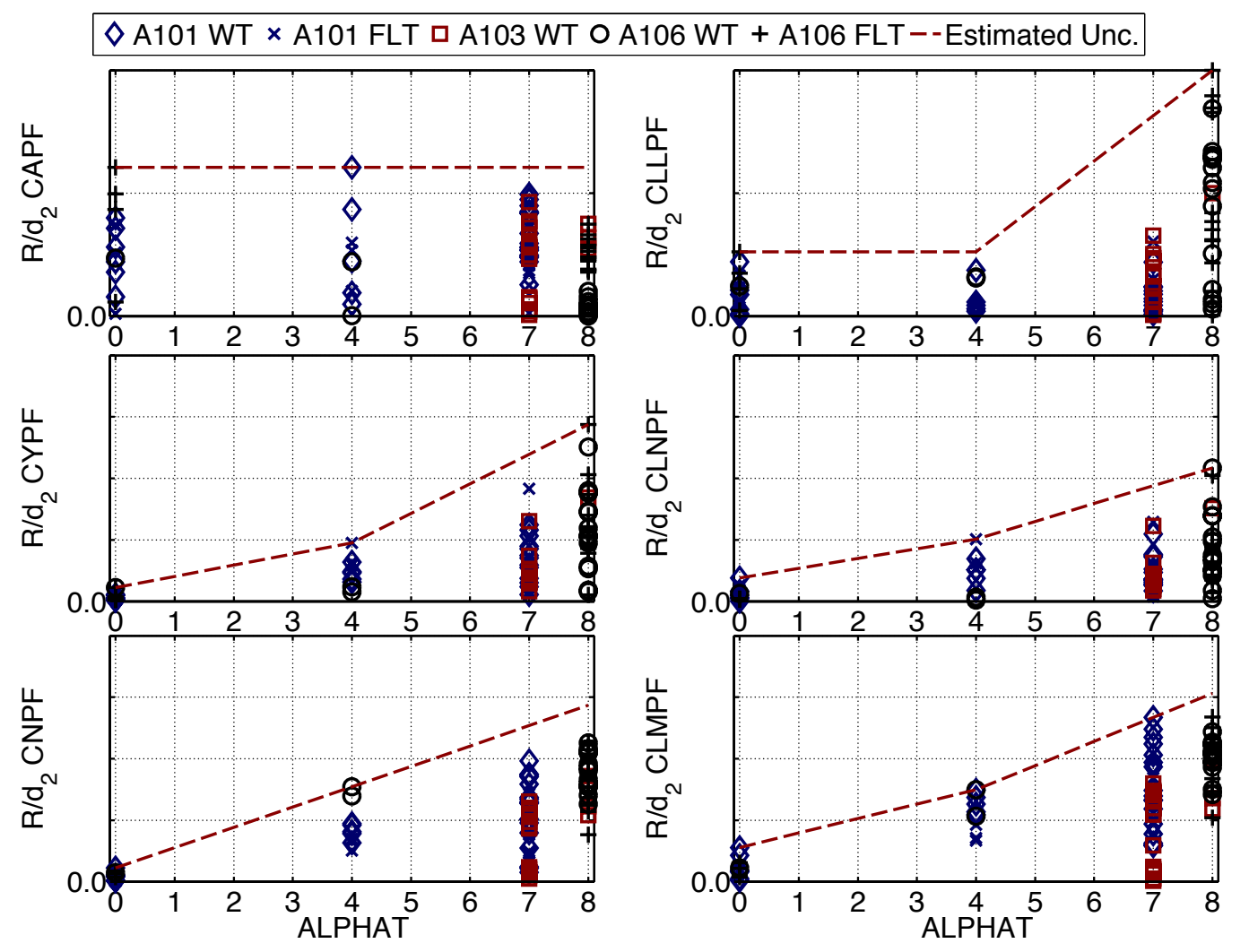

Figure 5. Normalized range data for code-to-code comparisons of Ares I CFD solutions for the A101, A103, and A106 OMLs at WT and FLT Re at $M \geq 1.6$ and estimated code-to-code uncertainties versus $\alpha_{T}$.

The other two terms in Eq. (3) arise from validation of the Ares I CFD data with experimental results. The Ares I aero team acquired the A106 CFD solutions prior to the wind tunnel test in accordance with Ares I CFD best practices, ${ }^{1}$ so the UQ team used computational and experimental data for a previous design, the A103 OML, to estimate the validation uncertainties. The A103 AFMA DB was based entirely on WT data for the A103 OML, with corrections made to remove experimental bias errors. Figure 6 shows a plot comparing the A103 CFD and AFMA DB data at $M=1.6$ and $\alpha_{T}=7$ degrees versus aerodynamic roll angle. In the figure, the CFD data are shown as red circles with the estimated code-to-code uncertainty shown as error bars, the DB data are shown as the blue solid line with diamond markers, and the shaded blue area bounded by the blue dashed lines represents the DB uncertainty intervals. ${ }^{24}$ For each available A103 CFD solution (74 total for $M \geq 1.6$ ), the UQ team computed a validation residual by subtracting the A103 AFMA DB data from the corresponding CFD data. The UQ team divided the CFD validation uncertainty into both a random and a bias term because the Ares aero team observed a consistent bias between the USM3D CFD solutions and WT data for the longitudinal coefficients over the course of the project (e.g., USM3D consistently predicted CAPF to be lower than the measured wind tunnel data). The UQ team concluded that the validation residuals depended primarily on the total angle of attack, so the team pooled the residuals for all Mach numbers and aerodynamic roll angles together at each total angle of attack. 


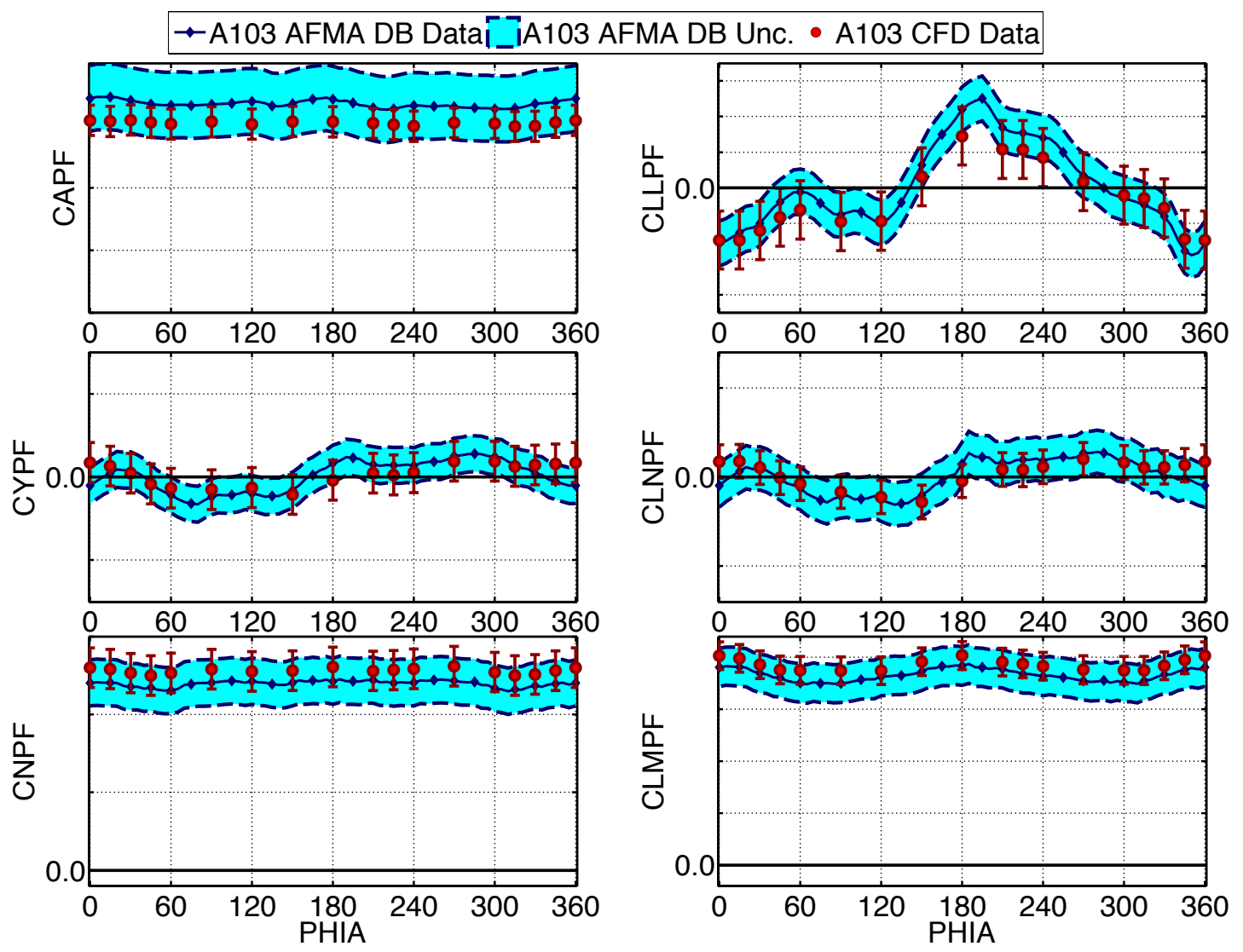

Figure 6. Comparison of A103 CFD data to the A103 AFMA DB at $M=1.6$ and $\alpha_{T}=7$ degrees versus $\phi_{a}$.

The UQ team estimated the bias term, denoted $E_{i, C F D, b i a s}$ in Eq. (3), by finding the median of the validation residuals at each total angle of attack and then making some slight adjustments using engineering judgment. The team assumed that the bias term was zero for the lateral-directional coefficients because the data did not suggest that a consistent bias existed across the entire domain. For the random component of the validation uncertainty, denoted $U_{i, \text { Val,random }}$ in Eq. (3), the UQ team subtracted the estimated bias uncertainty term from the validation residuals, resulting in a set of bias-corrected residuals. The team computed normalized ranges from the bias-corrected residuals and set the final estimate of the random uncertainty term equal to three times the mean normalized range. The team again made some minor adjustments to ensure smoothness. Figure 7 shows the A103 validation residuals (CFD minus WT DB data), the estimated bias uncertainty term (the solid purple line with square markers), and the random uncertainty term (the dotted purple bounds about the bias). The uncertainty intervals for the A103 AFMA $\mathrm{DB}^{24}$ are shown as dashed red lines in the figure for reference and are symmetric about zero since a validation residual equal to zero indicates no difference from the $\mathrm{DB}$ value. 


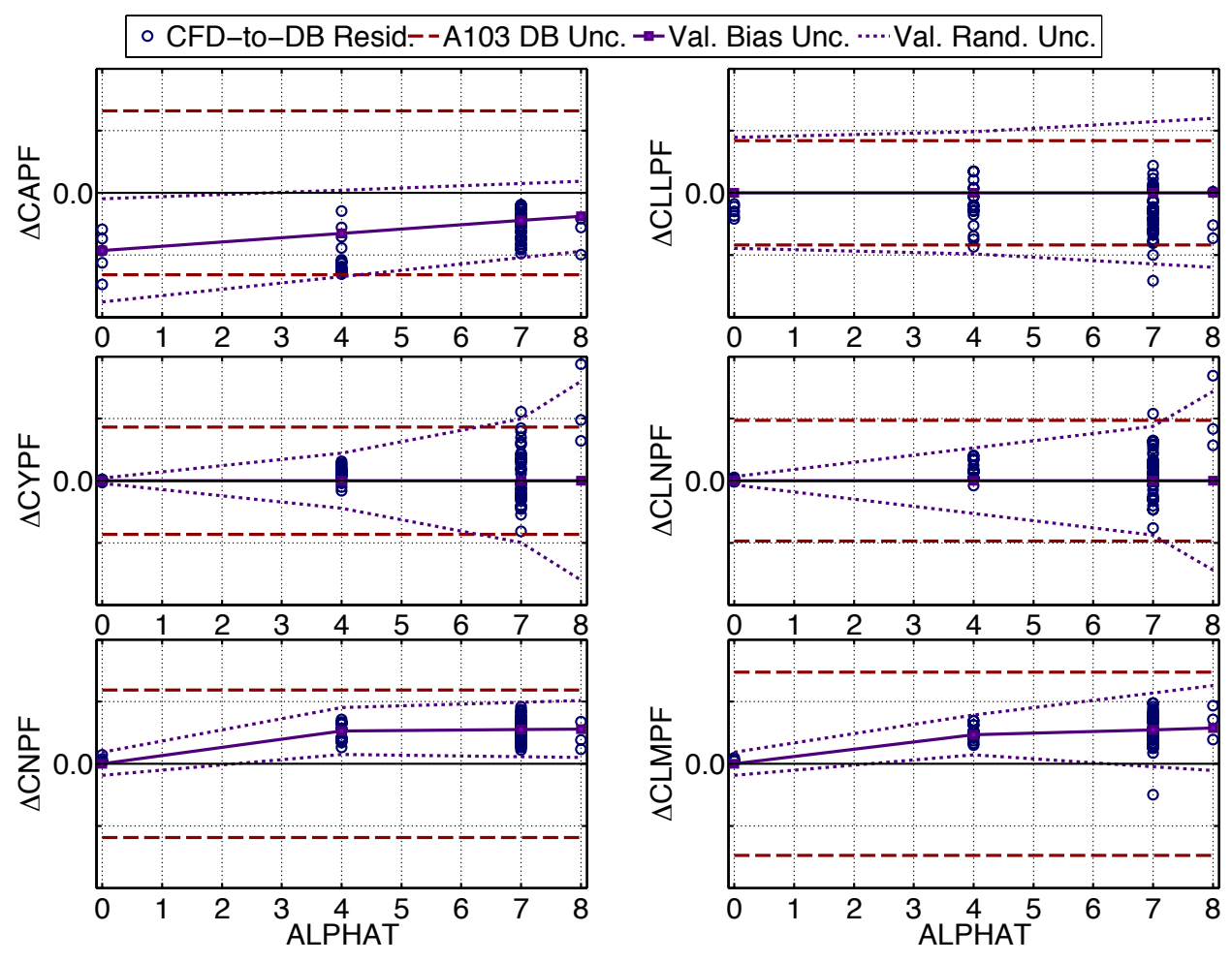

Figure 7. CFD-to-DB residuals for the A103 AFMA DB at $M \geq 1.6$ and estimated CFD validation bias and random uncertainties versus $\alpha_{T}$.

Table 4 lists the computed estimates for the code-to-code uncertainty and the bias and random terms of the CFD validation uncertainty at 8 degrees total angle of attack, reported as percentages of a reference value for each coefficient. The reference values for each coefficient are approximately the maximum magnitude for that coefficient across the entire A106 CFD-based AFMA DB. The results in Table 4 clearly show that the random uncertainties for the lateral-directional coefficients are much larger relative to the reference values than for the longitudinal coefficients. This is largely due to the fact that nominal DB values of the lateral-directional coefficients are much smaller than nominal values of the longitudinal coefficients. For instance, the normal force coefficient magnitudes are typically on the order of 10 times larger than the side force coefficient magnitudes at 8 degrees total angle of attack. The lateral-directional coefficients for the Ares I CLV are driven by complex phenomena such as vortex shedding, the interaction of wakes from various protuberances on other protuberances, and the vehicle boundary layer behavior; so the results in Table 4 are not surprising.

Table 4. CFD uncertainty estimates for the code-to-code, validation bias, and validation random terms as a percent of reference values at $\alpha_{T}=8$ degrees.

\begin{tabular}{|c|c|c|c|}
\hline Coefficient & Code-to-Code & Validation Bias & Validation Random \\
\hline$C_{A}$ & $0.96 \%$ & $-1.80 \%$ & $3.09 \%$ \\
\hline$C_{Y}$ & $31.9 \%$ & $0 \%$ & $154 \%$ \\
\hline$C_{N}$ & $2.39 \%$ & $3.50 \%$ & $3.30 \%$ \\
\hline$C_{l}$ & $13.3 \%$ & $0 \%$ & $41.4 \%$ \\
\hline$C_{m}$ & $1.82 \%$ & $2.57 \%$ & $3.52 \%$ \\
\hline$C_{n}$ & $28.9 \%$ & $0 \%$ & $165 \%$ \\
\hline
\end{tabular}

The UQ team also used a validation metric called the average relative error metric proposed in Ref. 21 to quantify the accuracy of the A103 CFD predictions relative to the corresponding A103 WT-based DB. This global metric is a measure of the average difference between the CFD and WT data relative to the WT data values at each point. The UQ team slightly modified the metric presented in Ref. 21. Since the lateral-directional coefficients for the Ares I CLV cross zero throughout the aerodynamic roll angle domain at a given total angle of attack, the UQ team did not use the mean DB data at each condition in the denominator of the metric as formulated in Ref. 21 . The 
team instead used the maximum A103 AFMA DB magnitude across the entire aerodynamic roll angle domain at each given Mach and total angle of attack. This formulation for the mean relative error metric is shown in Eq. (4), where $\hat{E}_{n}$ is the difference (or error) between the CFD and WT data for each solution, $C_{i, r e f, n}$ is the reference maximum value from the A103 AFMA DB for that condition, $N$ is the number of available CFD validation points, and $\left|\frac{\hat{E}}{C_{i, \text { ref }}}\right|_{\text {mean }}$ is the mean relative error metric.

$$
\left|\frac{\hat{E}}{C_{i, \text { ref }}}\right|_{\text {mean }}=\frac{1}{N} \sum_{n=1}^{N}\left|\frac{\hat{E}_{n}}{C_{i, \text { ref }, n}}\right|
$$

It is useful to compute median and maximum relative error metrics in addition to the mean relative error metric. The median relative error metric uses the same general equation above, and the maximum relative error metric corresponds to the maximum value of the term inside the summation on the right-hand-side of Eq. (4) at any of the CFD conditions. It is necessary to omit any points where the reference value is very close to zero to avoid artificially inflated the results of these metrics. For this computation, the UQ team omitted the points at 0 degrees total angle of attack for all coefficients except $C_{A}$ because the reference value magnitudes are very small for those conditions. To help interpret the significance of the mean relative error metric, the UQ team also normalized the WT DB uncertainty by the same reference data to compute a mean relative uncertainty (analogous to the mean relative confidence indicator in Ref. 21). Table 5 gives the mean, median, and maximum relative error metrics for the A103 CFD as well as the mean relative uncertainty. These metrics show that the relative errors for the CFD predictions of the lateral-directional coefficients are much higher than for the longitudinal coefficients, but the correspondingly higher mean relative uncertainties indicate that the WT DB uncertainties are also larger relative to the lateraldirectional coefficients. The large difference between the mean/median relative error and the maximum relative error metrics for the lateral-directional coefficients is also an indication that the CFD data may not adequately model certain behaviors in the WT data for those coefficients. The mean and median relative error metrics for all coefficients are less than the mean relative uncertainty from the A103 DB, but this is only a comparison of the mean values across the domain and does not indicate that the CFD-to-WT residuals are within the DB uncertainty intervals at any given point.

Table 5. Validation metrics for A103 CFD solutions and the mean relative A103 AFMA DB uncertainty.

\begin{tabular}{|c|c|c|c||c|}
\hline & \multicolumn{3}{|c|}{$\begin{array}{c}\text { CFD Validation Metrics } \\
\text { (Relative Error in Percent) }\end{array}$} & $\begin{array}{c}\text { Relative DB } \\
\text { Uncertainty }\end{array}$ \\
\hline Coefficient & Mean & Median & Maximum & Mean \\
\hline$C_{A}$ & $3.60 \%$ & $3.26 \%$ & $7.53 \%$ & $6.06 \%$ \\
\hline$C_{Y}$ & $27.8 \%$ & $26.1 \%$ & $94.9 \%$ & $94.9 \%$ \\
\hline$C_{N}$ & $6.26 \%$ & $6.00 \%$ & $13.2 \%$ & $11.9 \%$ \\
\hline$C_{l}$ & $17.0 \%$ & $14.3 \%$ & $66.6 \%$ & $38.6 \%$ \\
\hline$C_{m}$ & $4.09 \%$ & $4.17 \%$ & $8.50 \%$ & $10.4 \%$ \\
\hline$C_{n}$ & $24.4 \%$ & $20.4 \%$ & $97.0 \%$ & $86.0 \%$ \\
\hline
\end{tabular}

\section{B. Database Modeling Uncertainty}

The database modeling uncertainty for the CFD-based DB accounts for errors incurred by the process of taking the input CFD data and constructing the final DB. The UQ team evaluated if the NEAR RS output modeled the input CFD data by comparing the RS computed in NEAR RS to the input CFD data points. The prediction capability of the response surface was evaluated by comparing the RS computed in NEAR RS to the four CFD points obtained for database assessment in the domain that were not used to develop the DB. In both cases, the DBM uncertainties were only considered significant if the residuals between the RS and the actual CFD data fell outside of the estimated discretization error bounds of the CFD data, which were assumed to be the estimated code-to-code uncertainties.

The UQ team computed the range between the CFD-based DB values and the original CFD data at each of the 104 original WT Re CFD conditions and plotted the residuals versus total angle of attack. All of the residuals were within the estimated code-to-code uncertainty bounds. The same procedure was performed for the four DB

14

American Institute of Aeronautics and Astronautics 
assessment points, and again the UQ team observed that all of the residuals were within the estimated bounds. Therefore, no additional uncertainty for database modeling errors was included in the uncertainty model for the CFD-based DB because the observed database modeling errors were within the input data uncertainty intervals. Therefore, the UQ team set the $U_{i, D B M}$ term in Eq. (2) equal to zero.

\section{Database Interpolation Uncertainty}

The database interpolation uncertainty accounts for any errors that occur as a result of a database user querying the discrete database at any given condition within the domain. The implementation instructions for the CFD-based DB directed the user to linearly interpolate from the discrete database conditions to any condition in the domain, so the DBI uncertainty is directly related to the non-linearity of the data between the reported discrete conditions. To evaluate this uncertainty, the UQ team created a finely spaced set of query points across the domain and queried the final CFD-based DB at those points using both a linear interpolation and a higher-order cubic spline interpolation. The team evaluated the residuals between the two interpolation methods using the same methodology used for the DBM uncertainty. As with the DBM uncertainty, the UQ team observed that all of the residuals were within the estimated bounds and therefore set the $U_{i, D B I}$ term in Eq. (2) equal to zero.

\section{WT-based Supersonic A106 AFMA DB and Uncertainty}

The final WT-based Supersonic A106 AFMA DB developed from the UPWT Test 1972/1843 data provided the aerodynamic coefficients and uncertainties at the flight Reynolds number, using a wind-tunnel-to-flight Reynolds number correction derived from CFD. The full description of the development of the final A106 AFMA DB is provided in Ref. 26, while the details of the uncertainty analysis are presented in Ref. 10. This paper will briefly summarize the general procedures and highlight some modifications to the data made for the current comparisons, but the reader is directed to the reference documents for additional details. To ensure a valid comparison, this paper does not use the final WT-based Supersonic A106 AFMA DB as released, but instead uses the DB for the baseline A106 OML at WT Re developed directly from the UPWT Test 1972/1843 data.

\section{A. Database Development Summary}

The DB team constructed the WT-based DB for the baseline A106 OML using a procedure developed during the previous DACs (DAC-2A and DAC-2B) to remove systematic errors from the wind tunnel data. ${ }^{26}$ An axisymmetric model (designated the $\mathrm{C} 4$ configuration) of the A106 OML with all protuberances removed was tested in UPWT Test 1972/1843, and the DB team used this data to remove systematic errors associated with the wind tunnel from the baseline A106 configuration $(\mathrm{C} 1)$ data. Both the axisymmetric configuration and the baseline configuration were tested using pitch sweeps at fixed aerodynamic roll angles and roll sweeps at fixed total angles of attack. The overall construction of the WT-based DB for the baseline A106 OML is defined in Eq. (5).

$$
C_{i, D B}=\left(C_{i, C 1, p i t c h}-C_{i, C 4, \text { pitch }}\right)+C_{i, \mathrm{C} 4 \text { adjusted,pitch }}+\Delta C_{i, C 1, \text { roll }}
$$

The DB team first constructed "corrected" $C 4$ pitch sweep data (denoted $C_{i, C 4}$ adjusted,pitch) at 0 degrees aerodynamic roll angle by smoothing the $C 4$ WT pitch sweep data and invoking symmetry or anti-symmetry about 0 degrees angle of attack for the longitudinal coefficients $\left(C_{A}, C_{N}\right.$ and $\left.C_{m}\right)$, since an axisymmetric model would be expected to show symmetry (for $C_{A}$ ) or anti-symmetry (for $C_{N}$ and $C_{m}$ ) about 0 degrees. The lateral-directional coefficients $\left(C_{Y}\right.$, $C_{l}$ and $C_{n}$ ) for the "corrected" $C 4$ data were set to zero. Next, the DB team computed protuberance increments at 0 degrees aerodynamic roll angle by subtracting the measured $C 4$ pitch sweep data (denoted $C_{i, C 4, p i t c h}$ ) from the $C l$ pitch sweep data (denoted $C_{i, C l, p i t c h}$ ). Finally, the team calculated incremental coefficients due to aerodynamic roll angle dependence (denoted $\Delta C_{i, C l, \text { roll }}$ ) using the $C l \mathrm{WT}$ roll sweeps (available at total angles of attack of $0,2,4,6$, and 8 degrees) by subtracting the 0 degree values of the coefficients from the rest of the roll sweep. This process resulted in a database with independent variables of Mach number, total angle of attack, and aerodynamic roll angle.

\section{B. Uncertainty Modeling Summary}

The details of the uncertainty modeling for the baseline A106 AFMA DB are described in Ref. 10 and will not be repeated here. The overall uncertainty buildup for the DB at WT Re contains three terms: 1) the experimental uncertainty based on the wind tunnel repeatability, 2) the database modeling uncertainty for creating the baseline $\mathrm{DB}$, and 3) the database interpolation uncertainty. The experimental uncertainty for the WT-based DB presented in Ref. 10 depends only on the estimated data repeatability, which the UQ team computed using data from both the 
A106 test in the UPWT as well as data from previous Ares I WT tests in UPWT. Replicate runs were performed throughout each WT test, often with several days or significant configuration changes occurring between replicates, so the repeatability estimated by the UQ team could also be referred to as reproducibility based on the nomenclature of the US Guide to the Expression of Uncertainty in Measurement. ${ }^{27}$ The UQ team assumed that the DB development process corrected for any bias errors present in the WT data, and the estimated repeatability for the WT-based DB characterized all of the remaining variability in the wind tunnel environment. The final computed uncertainties for the A106 AFMA DB are much larger relative to the nominal data for the lateral-directional coefficients than for the longitudinal coefficients. However, the actual magnitude of the $C_{N}$ and $C_{Y}$ uncertainties are identical, and the same is true for $C_{m}$ and $C_{n}$. Throughout the Ares DAC process the UQ team generally found that the $C_{l}$ uncertainties were quite large relative to the coefficient values; however, that observation was not true for the WT-based Supersonic A106 AFMA DB uncertainty.

\section{Assessment of CFD-based Supersonic A106 AFMA DB}

The primary goal of this paper is to assess the quality of the CFD-based Supersonic A106 AFMA DB with respect to the final WT-based DB that was released. This assessment includes both a qualitative comparison of the general trends of the data with respect to the independent variables and a quantitative assessment of the values of the aerodynamic coefficients in both DBs. Since there are no formally defined requirements on the necessary accuracy of aerodynamic data for the Ares project, there is no way to definitively conclude whether the CFD-based DB is adequate relative to the WT-based DB. A general assessment will be made, however, by examining the qualitative comparisons of the data and the DB-to-DB residuals with respect to the computed uncertainty bounds.

There are striking qualitative similarities between the WT- and CFD-based DBs. Figure 8(a) shows an isometric surface plot of the WT-based DB for $C_{l}$ at 8 degrees total angle of attack versus Mach number and aerodynamic roll angle, corresponding to the same plot as Fig. 2 for the CFD-based DB. The CFD-based DB plot from Fig. 2 is repeated in Fig. 8(b) to aid in visually comparing the two DBs. Clearly, the general trends and behaviors of the DBs are very similar across the domain shown. This general qualitative similarity is true for all other aerodynamic coefficients in both DBs. 


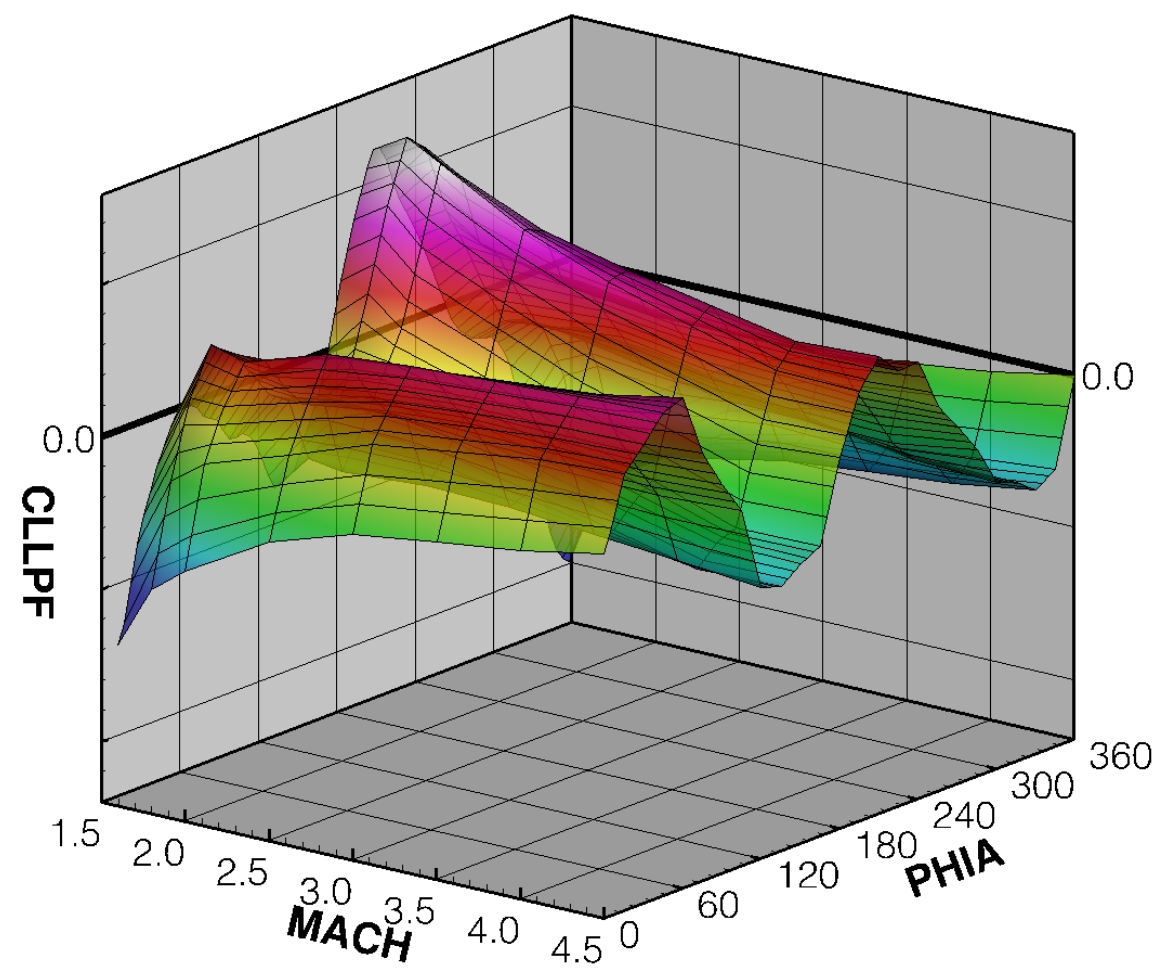

Figure 8(a). WT-based A106 AFMA DB for $C_{l}$ at $\alpha_{T}=8$ degrees versus $M$ and $\phi_{a}$.

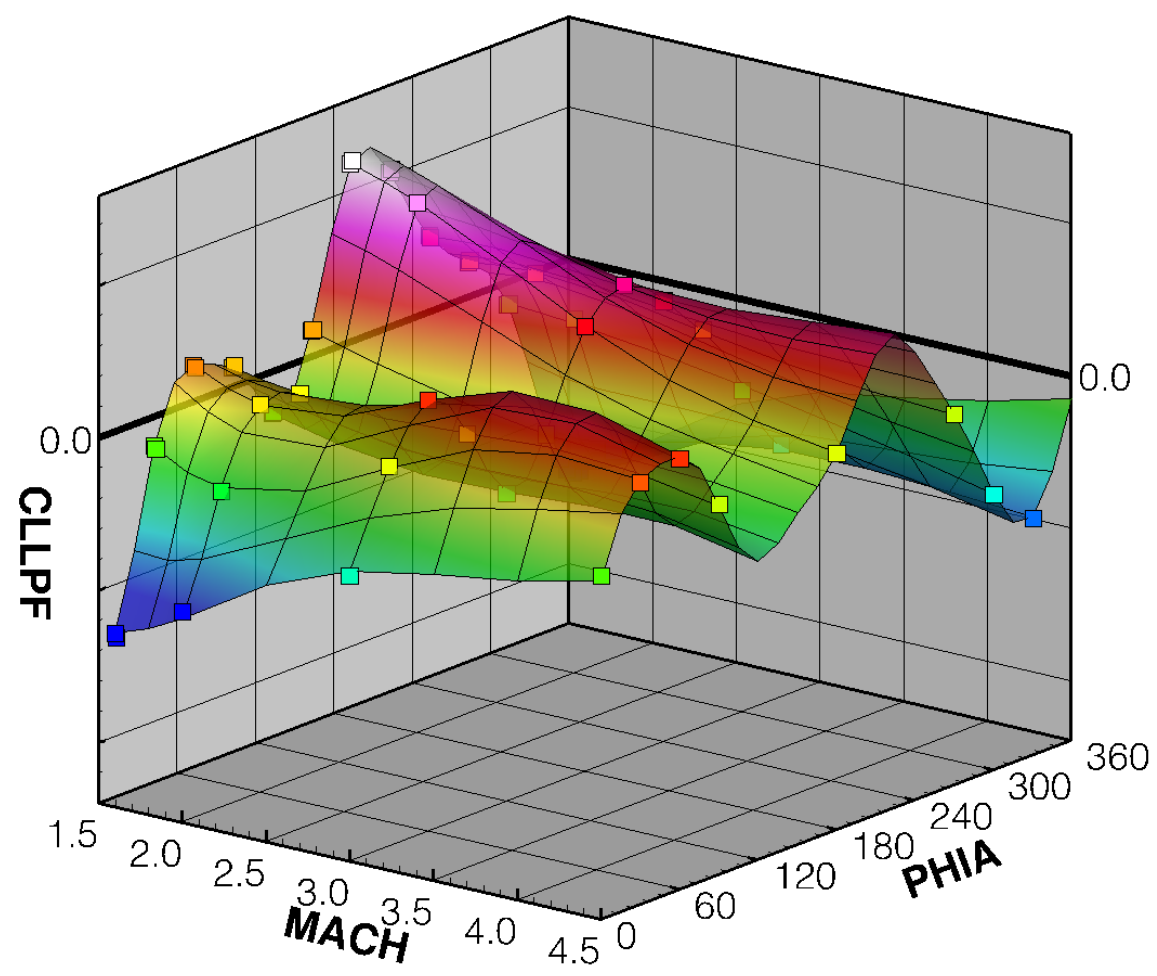

Figure 8(b). CFD-based Supersonic A106 AFMA DB and WT Re CFD data for $C_{l}$ at $\alpha_{T}=8$ degrees versus $M$ and $\phi_{a}$ (repeated from Fig. 2 for comparison). 
Figure 9 shows line plots of the CFD-based and WT-based DBs versus total angle of attack at Mach 3.0 and an aerodynamic roll angle of 90 degrees, while Fig. 10 shows the same comparison at Mach 2.0 and 285 degrees aerodynamic roll. These two conditions were indicative of the best and worst qualitative comparisons, respectively, of the DB trends with respect to total angle of attack. For all coefficients except $C_{Y}$ and $C_{n}$, it is clear that both DBs exhibit very similar behavior with respect to total angle of attack in both cases. However, for the side force and yawing moment coefficients, Fig. 10 shows that at certain conditions the trends diverge between the databases, but generally the agreement is reasonable. Figure 11 compares the DBs at 8 degrees total angle of attack at Mach 1.6 versus aerodynamic roll angle and clearly shows that the WT-based DB $C_{Y}$ and $C_{n}$ data exhibit more oscillatory behavior versus aerodynamic roll angle than the CFD-based DB data. This is generally true throughout the DB. Additionally, Fig. 11 shows that while the $C_{l}$ trends in the two DBs are similar, there are noticeable differences between the DBs, especially for aerodynamic roll angles above about 240 degrees. In all of the qualitative comparison plots, the black dotted lines with circle markers denote the CFD-based DB, the shaded grey regions bounded by dashed black lines show the CFD-based DB uncertainties, the solid green lines with square symbols depict the WT-based DB, and the green shaded areas bounded by green dash-dot lines are the WT-based DB uncertainties. Each grid section in the figures is the same size for $C_{N}$ and $C_{Y}$ and $C_{m}$ and $C_{n}$.

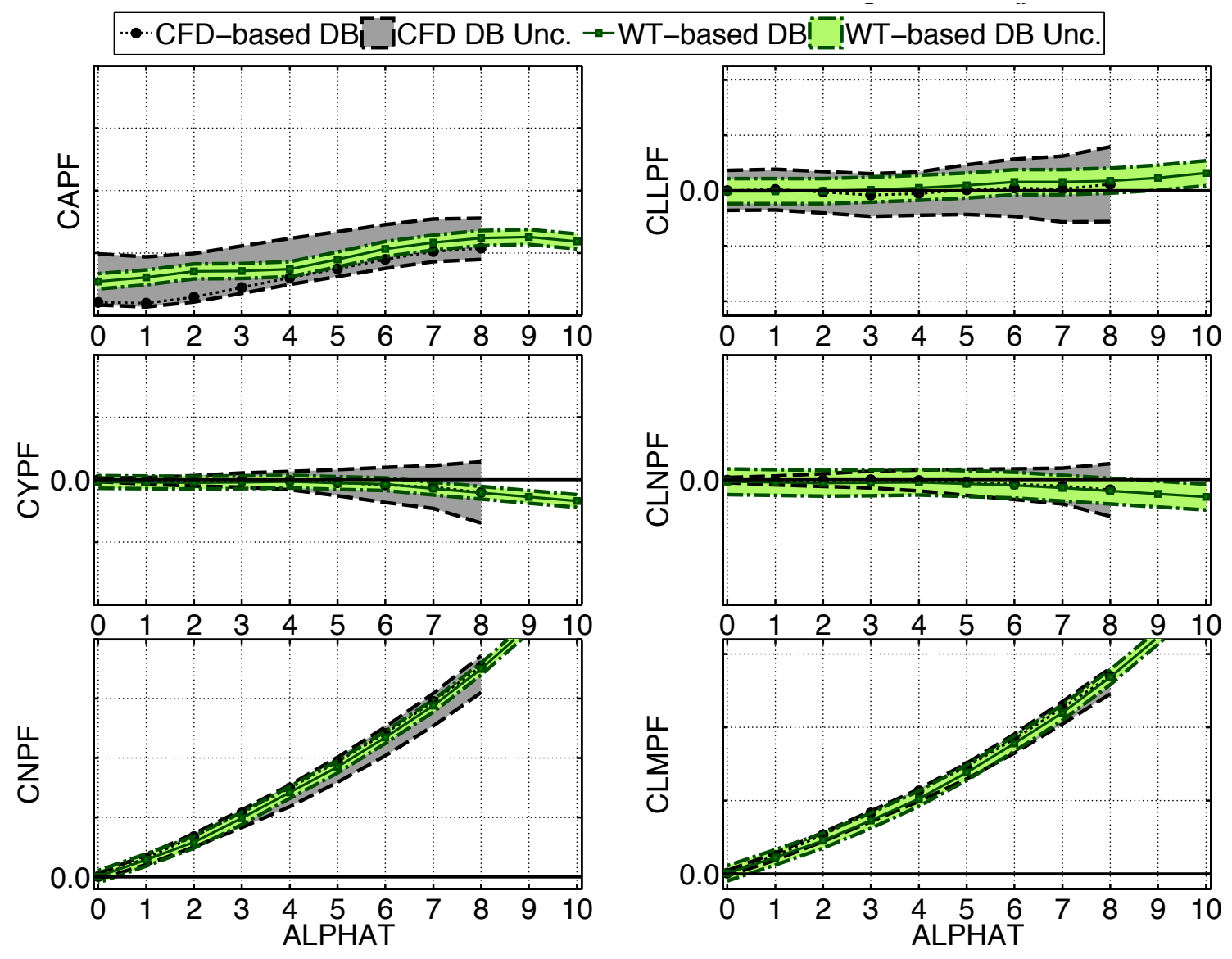

Figure 9. Comparison of CFD-based and WT-based A106 AFMA DBs and uncertainty bounds at $M=3.0$ and $\phi_{a}=90$ degrees versus $\alpha_{T}$. 


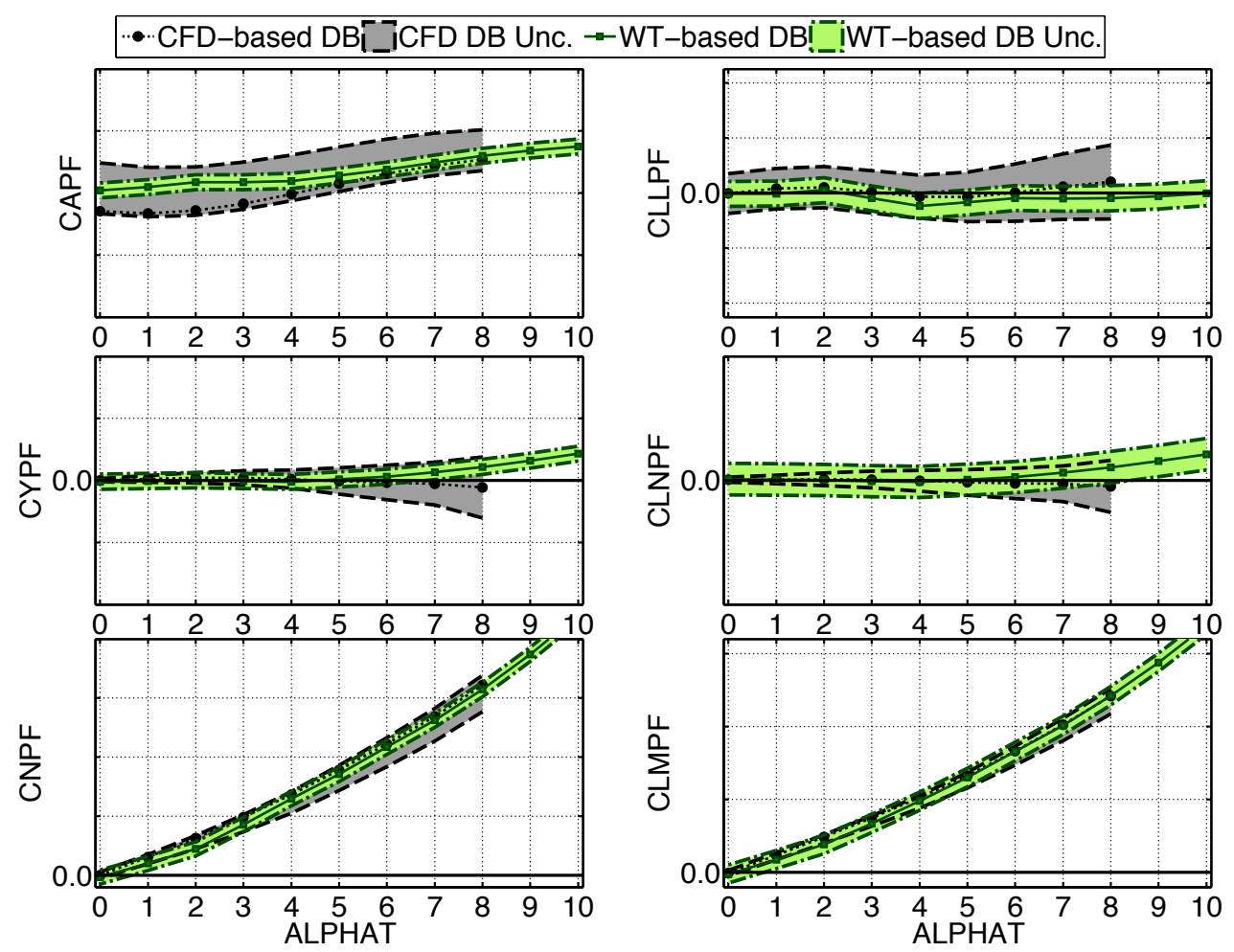

Figure 10. Comparison of CFD-based and WT-based A106 AFMA DBs and uncertainty bounds at $M=$ 2.0 and $\phi_{a}=285$ degrees versus $\alpha_{T}$.

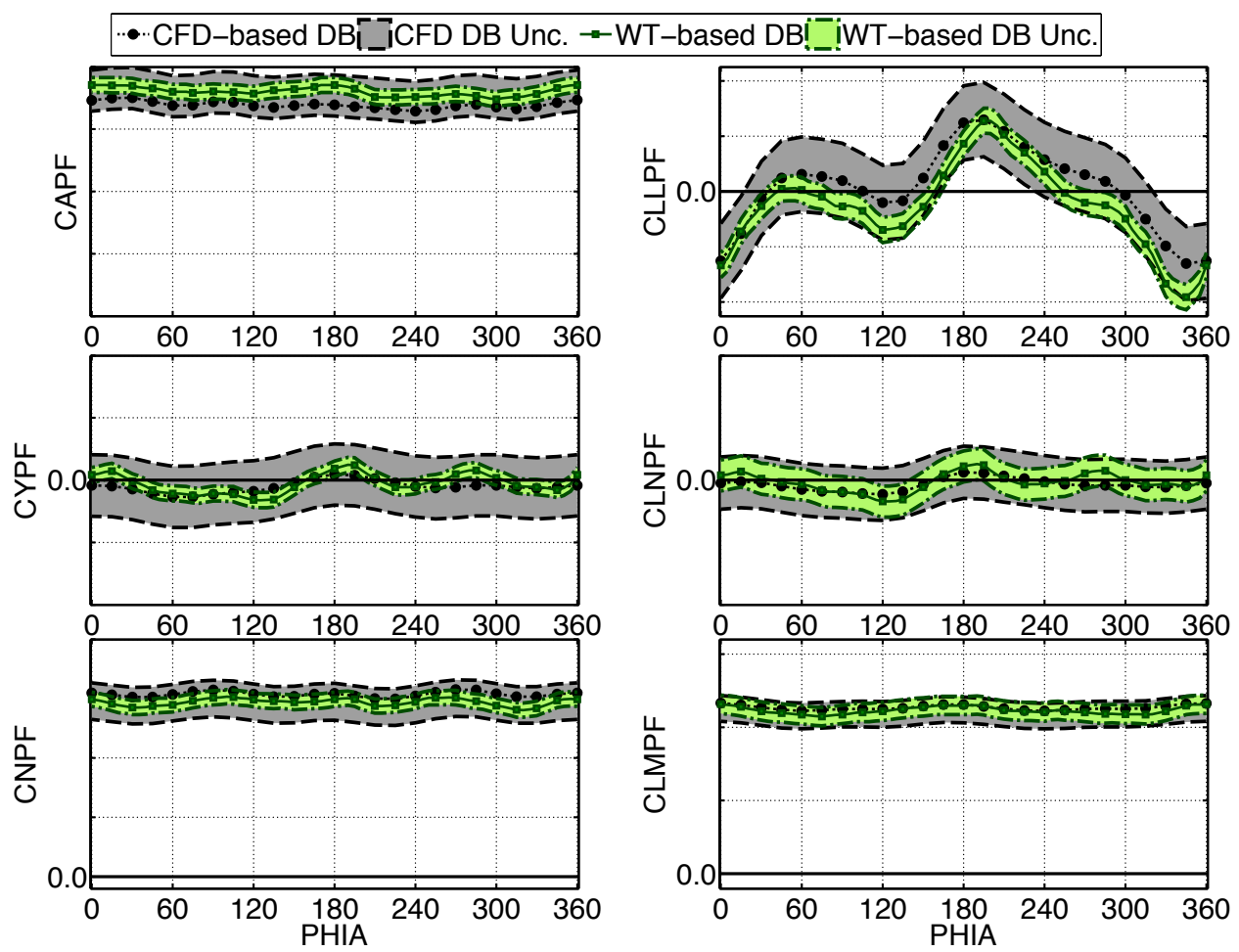

Figure 11. Comparison of CFD-based and WT-based A106 AFMA DBs and uncertainty bounds at $M=$ 1.6 and $\alpha_{T}=8$ degrees versus $\phi_{a}$. 
The comparison plots in Fig. 9-11 also illustrate the differences in the uncertainties for the two DBs. The uncertainties for the WT-based DB depend only on Mach number and change little throughout the entire domain, while Fig. 9 and 10 clearly show that the CFD-based DB uncertainties vary with total angle of attack, especially for $C_{Y}$ and $C_{n}$. This is due mostly to the differences in the input data uncertainties; the experimental uncertainty term in the WT-based DB uncertainty is based solely on the experimental repeatability, which was estimated as a single value for each Mach number. The CFD-based DB input data uncertainty is based on the code-to-code comparisons and validation comparisons, both of which are much smaller near zero degrees than for higher values of total angle of attack. The estimated CFD-based DB uncertainties are larger than the WT-based DB uncertainties at the higher total angles of attack for all coefficients. However, at zero degrees total angle of attack, the CFD-based DB uncertainties are actually smaller than the WT-based DB estimates for all coefficients except $C_{A}$ and $C_{l}$; for those two coefficients, the CFD-based DB uncertainties are larger throughout the domain. Although it is not clearly visible in Fig. 9-11, the CFD-based DB uncertainties are offset from the nominal DB values for the longitudinal coefficients due to the bias term from the CFD validation uncertainty, while the WT-based DB uncertainties are symmetric about the nominal DB. An alternative to presenting this offset uncertainty would be to add the bias term from the CFD validation comparison to the nominal CFD-based DB itself, which would result in symmetric bounds.

Perhaps the most important metric for assessing the adequacy of the CFD-based DB is to quantify whether the true values of the aerodynamic data at WT Reynolds number are contained by the estimated uncertainty intervals. However, since the true values cannot be known, the UQ team instead evaluated whether the values predicted by the WT-based DB are contained by the uncertainty intervals estimated for the CFD-based DB. The UQ team plotted the DB-to-DB residuals (CFD-based DB minus WT-based DB) and then compared the residuals to the estimated uncertainty bounds. Figure 12 shows plots of the DB-to-DB residuals against total angle of attack along with the CFD-based DB uncertainty intervals shown as red dashed lines. It is clear from the figure that a large majority of the residuals are contained by the estimated uncertainties. Table 6 reports the percentage of the DB points that fall within the estimated CFD-based DB uncertainty intervals for each coefficient. For all coefficients except $C_{Y}$, at least 98.4 percent of the DB-to-DB residuals fall within the uncertainty intervals, but 92.3 percent of the $C_{Y}$ residuals still fell within the estimated CFD-based uncertainty intervals. Furthermore, Fig. 12 shows that the residuals that do fall outside of the CFD-based DB uncertainty intervals are generally quite close to the bounds of the uncertainty. Since the WT-based DB values are also uncertain, it is useful to also compare the DB-to-DB residuals to a combined uncertainty. Table 6 shows that comparing the residuals to a combined uncertainty computed by root-sum-squaring the random component of the CFD-based DB uncertainty and the WT-based DB uncertainty increases the percentage of points within the bounds to 100 percent for all coefficients except rolling moment, where the percent within the bounds is 99.6 percent. Both of these metrics indicate that the CFD-based DB and estimated uncertainties do an excellent job of predicting the actual aerodynamic coefficients of the WT-based DB, which was the original purpose of the CFD-based DB.

Table 6. Percentage of DB-to-DB residuals for the CFD-based and WT-based DBs within the uncertainty intervals estimated for the CFD-based DB and for a combined DB uncertainty.

\begin{tabular}{|c|c|c|}
\hline & \multicolumn{2}{|c|}{ Percentage of DB-to-DB Residuals Within Interval } \\
\hline Coefficient & CFD-based DB Uncertainty & Combined Uncertainty \\
\hline$C_{A}$ & $100 \%$ & $100 \%$ \\
\hline$C_{Y}$ & $92.3 \%$ & $100 \%$ \\
\hline$C_{N}$ & $99.9 \%$ & $100 \%$ \\
\hline$C_{l}$ & $99.4 \%$ & $99.6 \%$ \\
\hline$C_{m}$ & $98.4 \%$ & $100 \%$ \\
\hline$C_{n}$ & $99.4 \%$ & $100 \%$ \\
\hline
\end{tabular}




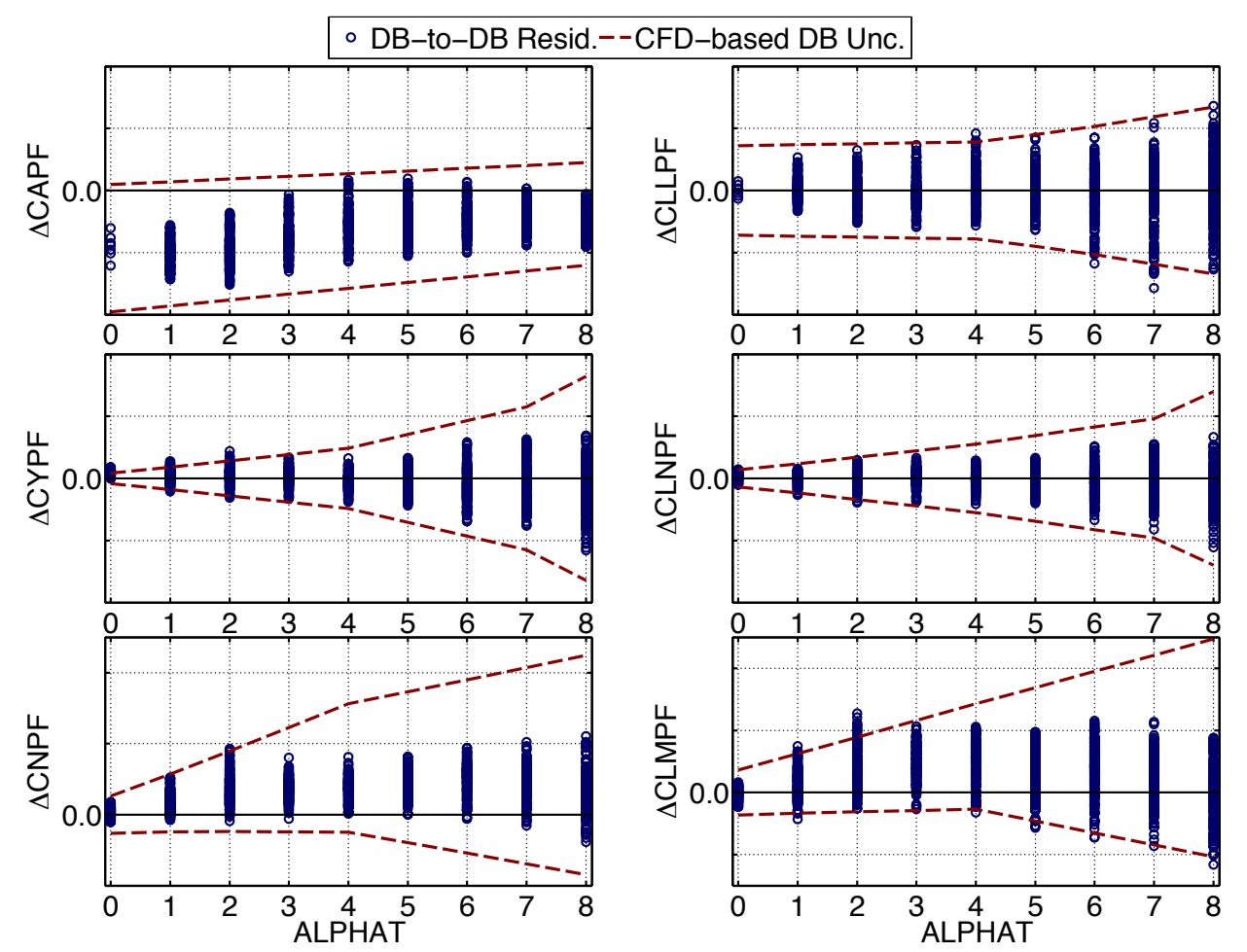

Figure 12. DB-to-DB residuals for the CFD-based and WT-based Supersonic A106 AFMA DBs and the estimated CFD-based uncertainty intervals versus $\alpha_{T}$.

Another way to quantify the differences between the two databases is to apply the validation metrics discussed earlier, such as the mean relative error metric, to the DB-to-DB residuals. Table 7 shows the mean, median, and maximum relative error metrics for the DB comparison as well as the mean relative uncertainty computed using the combined uncertainty discussed above. As for the CFD validation computations, the reference values for each coefficient are the maximum magnitude of the CFD-based DB at the given Mach number and total angle of attack and conditions, and the points at 0 degrees total angle of attack were omitted for all coefficients except $C_{A}$. The relative errors for the lateral-directional coefficients are quite large, especially the maximum relative error. As noted for the CFD validation comparison, the large values of the relative error and mean relative uncertainty for the lateral-directional coefficients is primarily because the reference values for those coefficients are quite small throughout the database. The mean magnitudes of the DB-to-DB residuals themselves are actually smaller for $C_{Y}$ and $C_{n}$ than for $C_{N}$ and $C_{m}$, respectively.

Table 7. Relative DB-to-DB error metrics for the CFD-based and WT-based A106 AFMA DBs and the mean relative combined uncertainty.

\begin{tabular}{|c|c|c|c||c|}
\hline & \multicolumn{3}{|c||}{$\begin{array}{c}\text { DB-to-DB Relative Error Metrics } \\
\text { (Relative Error in Percent) }\end{array}$} & $\begin{array}{c}\text { Relative } \\
\text { Uncertainty }\end{array}$ \\
\hline Coefficient & Mean & Median & Maximum & Mean \\
\hline$C_{A}$ & $4.50 \%$ & $4.44 \%$ & $10.3 \%$ & $6.65 \%$ \\
\hline$C_{Y}$ & $51.6 \%$ & $40.1 \%$ & $326 \%$ & $238 \%$ \\
\hline$C_{N}$ & $5.75 \%$ & $4.92 \%$ & $30.2 \%$ & $16.7 \%$ \\
\hline$C_{l}$ & $33.2 \%$ & $24.1 \%$ & $187 \%$ & $158 \%$ \\
\hline$C_{m}$ & $4.16 \%$ & $3.02 \%$ & $28.2 \%$ & $16.4 \%$ \\
\hline$C_{n}$ & $50.0 \%$ & $37.0 \%$ & $442 \%$ & $389 \%$ \\
\hline
\end{tabular}

It is also important to evaluate whether the CFD validation uncertainty estimates developed by the UQ team from the A103 CFD data do an adequate job of modeling the differences between the A106 CFD and WT data. This postfacto comparison was not possible at the time the CFD-based DB was released because the WT data for A106 were 
not available at that time. However, this evaluation is an important step in confirming the adequacy of the uncertainty model for the CFD-based DB presented in this paper. Figure 13 shows the A106 CFD data (as red circles with error bars from the code-to-code uncertainty term) and the A106 WT-based DB (green lines with square markers) and uncertainty intervals (shaded green regions bounded by dash-dot lines) versus aerodynamic roll angle at the same conditions shown for the A103 CFD in Fig. 6 (Mach 1.6 at a total angle of attack of 7 degrees). The qualitative differences between the CFD and WT DB for A103 and A106 appear to be very similar when comparing Fig. 13 to Fig. 6. Figure 14 shows the A106 CFD validation residuals (CFD data minus WT-based DB values) in comparison to the estimated A103 validation uncertainties. The estimated A103 validation bias term is shown as a dashed purple line and the estimated A103 validation random term bounds are shown as purple dotted lines that are symmetric about the bias. The estimated A103 uncertainty bounds contain most of the A106 residuals, and the estimated A103 validation biases appear reasonable for the A106 data as well. There are a few A106 residuals outside of the A103 uncertainty estimates, especially for $C_{Y}$ and $C_{n}$ near 0 degrees and $C_{N}$ and $C_{m}$ above 4 degrees total angle of attack. The good general agreement between the A103 estimates and the A106 data confirms that the relationship between the CFD and WT data did not change significantly between design cycles for this data set, and this analysis provides confidence in the general methodology of using validation comparisons from previous design cycles to estimate validation uncertainty for the Ares I Supersonic AFMA DB. The excellent agreement between the validation biases for A103 and A106 suggests that instead of including the bias term in the uncertainty, the DB team could have applied the estimated biases as a correction to the CFD-based DB itself.

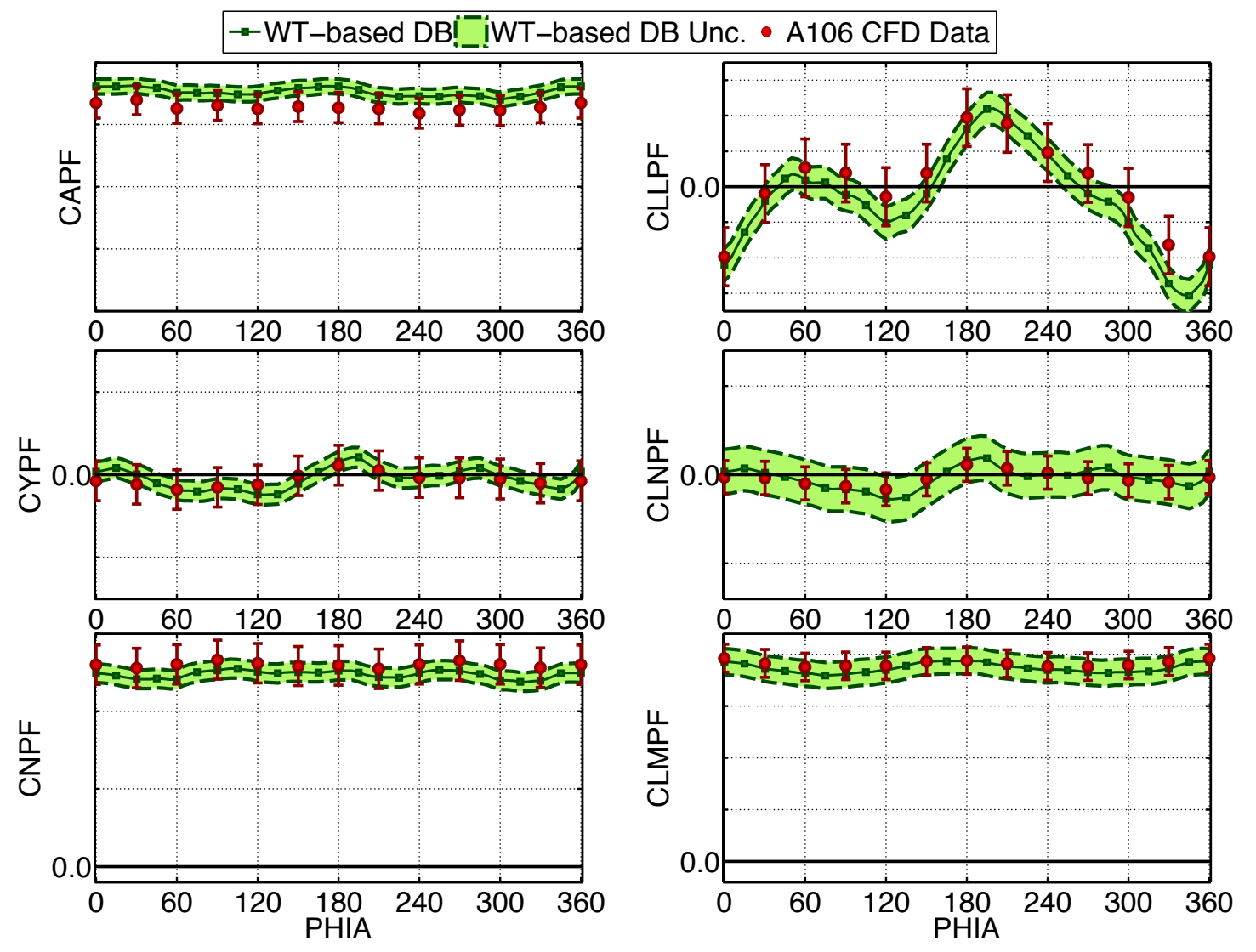

Figure 13. Comparison of A106 CFD data to UPWT data and the WT-based DB uncertainty bounds at $M=1.6$ and $\alpha_{T}=7$ degrees versus $\phi_{a}$. 

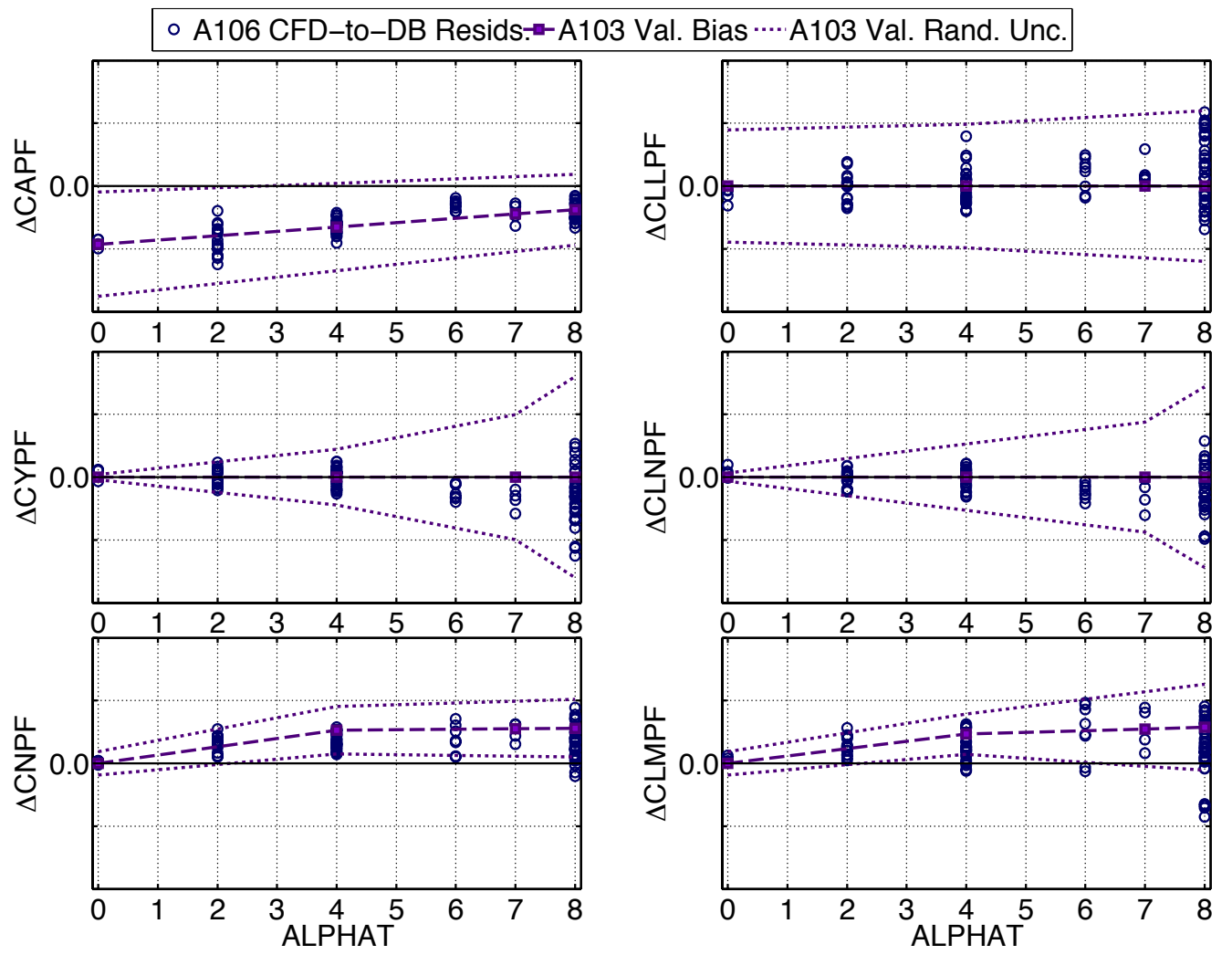

Figure 14. A106 CFD-to-WT residuals for the A106 CFD data at $M \geq 1.6$ and estimated A103 CFD validation bias and random uncertainties versus $\alpha_{T}$.

\section{Conclusions}

Although the CFD-based and WT-based Supersonic A106 AFMA DBs were developed independently and the input CFD and UPWT data were not adjusted in any way, the final resulting DBs exhibit qualitatively similar behavior throughout most of the domain, especially for the longitudinal aerodynamic coefficients. The two DBs do show diverging results for $C_{Y}$ and $C_{n}$ at some conditions, but the majority of the differences are within the uncertainty intervals for the CFD-based DB. The intended purpose of the CFD-based DB was to predict the aerodynamic coefficients in the WT-based DB before the WT data was available, and the quantitative comparison between the DBs suggests that the CFD-based DB did an admirable job at this purpose. Although the uncertainty intervals for the CFD-based $\mathrm{DB}$ presented here were not provided at the time of release because of schedule constraints and the methodology's immaturity at the time, all of the data needed to estimate the CFD-based DB uncertainties were available at the time of release. The estimated uncertainties for the two DBs are quite different in character, and the CFD-based DB uncertainties are generally larger than the WT-based DB except at low total angles of attack.

The assessment of the CFD-based DB presented in this paper suggests that the methods used by the Ares aero team both in acquiring the A106 CFD data and developing the CFD-based DB produced reliable and reasonably accurate results given the estimated uncertainty intervals. This is significant because at the time of the release of the CFD-based DB, there was considerable concern as to whether CFD could be used without the use of any WT data to construct such a DB for the Ares I CLV. The current analysis shows that the methodology used to develop the CFD-based DB could be used in future applications, provided that certain information about the CFD analyses and general behavior of the data are available and the CFD test matrix and DB development strategy are designed with response surface generation in mind. The DB team found that knowledge of the behavior of the earlier Supersonic A103 AFMA DB was critical to the development of the CFD-based DB for A106 because the team needed that knowledge in order to properly fill gaps in the CFD test matrix and ensure reasonable behavior of the aerodynamic coefficients. Additionally, it was essential that the Ares aero team conducted the A106 CFD simulations by following an established set of best practices that were also used for the earlier A103 CFD simulations. This 
continuity in the analysis methodology allowed the UQ team to assume that the A103 validation estimates could be used for the A106 OML. The present analysis confirmed that this assumption was reasonable because the validation residuals for both the A103 and A106 OMLs were similar.

\section{Acknowledgments}

The author would like to thank Khaled Abdol-Hamid for providing the USM3D CFD data for the A106 OML and Jing Pei and Heather Houlden for providing the data for the final A106 AFMA DB. Additional thanks are due to Mike Hemsch, Eric Walker, Amber Favaregh, Jeremy Pinier, and Heather Houlden for their aid in the development of the overall UQ methodology for the A106 AFMA DBs and for discussions and advice regarding the current analysis. Finally, the author thanks Kay Forrest and Steve Bauer for their help in editing this manuscript.

\section{References}

${ }^{1}$ Pao, S. P., Vatsa, V. N., Abdol-Hamid, K. S., Pirzadeh, S., Samareh, J. A., Klopfer, G. H., Taft, J. R., and Parlette, E. B., "Best Practice for Ascent Aerodynamics Analysis for the Ares I Configurations," Proceedings of the 55 $5^{\text {th }}$ JANNAF Propulsion Meeting, CPIA Publication, Boston, MA. May 12-16, 2008.

${ }^{2}$ Erickson, G. E., "0.01-Scale Ares I ADAC-3 (A106) Force and Moment Model Testing in the NASA Langley Research Center Unitary Plan Wind Tunnel," NASA Ares-AD-TA-0018, June 21, 2010.

${ }^{3}$ Hall, R. M., Holland, S. D., and Blevins, J. A., "Aerodynamic Characterization Overview of a Modern Launch Vehicle," AIAA-2011-10, January 2011.

${ }^{4}$ Tomek, W. G., Erickson, G. E., Pinier, J. T., and Hanke, J. L., "Overview of Experimental Investigations for Ares I Launch Vehicle Development," AIAA-2011-13, January 2011.

${ }^{5}$ Pinier, J. T., Niskey, C. J., Hanke, J. L., and Tomek, W. G., “Ares I Aerodynamic Testing at the Boeing Polysonic Wind Tunnel," AIAA-2011-998, January 2011.

${ }^{6}$ Abdol-Hamid, K. S., Ghaffari, F. and Parlette, E. B., "Overview of Ares I CFD Ascent Aerodynamic Data Development And Analysis Based on USM3D," AIAA-2011-15, January 2011.

${ }^{7}$ Pao, S. P., Deere, K. A., and Abdol-Hamid, K. S., "Establishing Approaches to Modeling the Ares I-X and Ares I Roll Control System with Free-stream Interaction", AIAA-2011-1056, January 2011.

${ }^{8}$ Pandya, M. J., Frink, N. T., Abdol-Hamid, K. S., Samareh, J. A., Parlette, E. B., and Taft, J. T., "Enhancements to NASA TetrUSS for Constellation Program,” AIAA-2011-1111, January 2011.

${ }^{9}$ Klopfer, G. H., Onufer, J. T., Chan, W. N., Pandya, S. A., Kless, J., and Lee, H. C., "Analysis of the Ares I Launch Vehicle Stage Separation: Static, Time Accurate, Prescribed Motion, and Coupled 6-DOF Simulations," To be published at the 49th AIAA Aerospace Sciences Meeting, Orlando 2011.

${ }^{10}$ Houlden, H. P, Favaregh, A. L., and Hemsch, M. J., "Quantification of the Uncertainties for the Ares I A106 Ascent Aerodynamic Database,” AIAA-2010-4926, June 2010.

${ }^{11}$ Frink, N. T., "Tetrahedral Unstructured Navier-Stokes Method for Turbulent Flows", AIAA Journal, Vol. 36, No. 11, November 1998, pp. 1975-1982.

${ }^{12}$ Buning, P. G., Chan, W. M., Renze, K. J., Sondak, D.L., Chiu, I. T., and Slotnick, J.P., OVERFLOW User's Manual, Version 1.6ab, NASA Ames Research Center, Moffett Field, CA, Jan. 1993.

${ }^{13}$ Hanke, J. L., "Uncertainty Quantification Efforts for CFD Results of the NASA Ares I Design Analysis Cycle 2 (ADAC2) Configurations," Proceedings of the 55th JANNAF Propulsion Meeting, CPIA Publication, Boston, MA. May 12-16, 2008.

${ }^{14}$ Mullur, A. A. and Messac, A., "Metamodeling using extended radial basis functions: a comparative approach," Engineering with Computers, Vol. 21, December 9, 2005, pp. 203-217.

${ }^{15}$ Meckesheimer, M., Booker, A. J., Barton, R. R., and Simpson, T. W., "Computationally Inexpensive Metamodel Assessment Strategies," AIAA Journal, Vol. 40, No. 10, October 2002, pp. 2053-2060.

${ }^{16}$ Reisenthel, P. H., Love, J. F., Lesieutre, D. J., and Childs, R. E., "Cumulative global metamodels with uncertainty -- a tool for aerospace integration," The Aeronautical Journal, Vol. 110, June 2006, pp. 375-384.

${ }^{17}$ Reisenthel, P. H., Love, J. F., Lesieutre, D. J., and Dillenius, M. F. E. D., "Innovative Fusion of Experiment and Analysis for Missile Design and Flight Simulation," Paper 23, Proceedings of the RTO AVT-135 Symposium on Innovative Missile Systems, Amsterdam, The Netherlands, May 15-19, 2006.

${ }^{18}$ Reisenthel, P. H., Love, J. F., Lesieutre, D. J., and Childs, R. E., "Cumulative Global Metamodels with Uncertainty: a Tool for Aerospace Integration," NEAR Paper 403, CEIAT 2005-0019, 1st International Conference on Innovation and Integration in Aerospace Sciences, Queen's University, Belfast, Northern Ireland, UK, 4-5 Aug. 2005.

${ }^{19}$ Rodman, L. C., Reisenthel, P. H., and Childs, R. E., "An Automated Documentation and Reporting System For CFD," AIAA 2002-0986, Jan. 2002.

${ }^{20}$ Roache, P. J, Verification and Validation in Computational Science and Engineering, Hermosa Publishers, Albuquerque, NM, 1998.

${ }^{21}$ Oberkampf, W. L. and Roy, C. J., Verification and Validation in Scientific Computing, Cambridge University Press, Cambridge, U. K., 2010.

${ }^{22}$ Guide for the Verification and Validation of Computational Fluid Dynamics Simulations, American Institute of Aeronautics and Astronautics, AIAA-G-077-1998, 1998. 
${ }^{23}$ Wheeler, D. J., Understanding Industrial Experimentation, SPC Press Inc., Knoxville, TN, 1990.

${ }^{24}$ Hemsch, M. J., Hanke, J. L., Walker, E. L., and Houlden, H. P., "Detailed Uncertainty Analysis for Ares I Ascent Aerodynamics Wind Tunnel Database," AIAA-2008-4259, June 2008.

${ }^{25}$ Wheeler, A. J., and Ganji, A. R., Introduction to Engineering Experimentation, $2^{\text {nd }}$ ed., Pearson Education, Inc., Upper Saddle River, NJ, 2004.

${ }^{26}$ Pamadi, B. N., Pei, J., Covell, P. F., Favaregh, N. M., Gumbert, C. G., and Hanke, J. L., “Aerodynamic Analyses and Database Development for Lift-Off/Transition and First Stage Ascent of the Ares I A106 Vehicle," AIAA-2011-12, January 2011.

${ }^{27}$ U.S. Guide to Expression of Uncertainty in Measurement, American National Standards Institute, ANSI/NCSL Z540.21997 (R2002). 